\title{
Article \\ Base Point Freeness, Uniqueness of Decompositions and Double Points for Veronese and Segre Varieties
}

\author{
Edoardo Ballico
}

check for

updates

Citation: Ballico, E. Base Point Freeness, Uniqueness of Decompositions and Double Points for Veronese and Segre Varieties. Symmetry 2021, 13, 2344. https:/ / doi.org/10.3390/sym13122344

Academic Editor: Rafał Zdunek

Received: 12 November 2021 Accepted: 1 December 2021 Published: 6 December 2021

Publisher's Note: MDPI stays neutral with regard to jurisdictional claims in published maps and institutional affiliations.

Copyright: (C) 2021 by the author. Licensee MDPI, Basel, Switzerland. This article is an open access article distributed under the terms and conditions of the Creative Commons Attribution (CC BY) license (https:/ / creativecommons.org/licenses/by/ $4.0 /)$.
Department of Mathematics, University of Trento, 38123 Trento, TN, Italy; edoardo.ballico@unitn.it

\begin{abstract}
We prove a base point freeness result for linear systems of forms vanishing at general double points of the projective plane. For tensors we study the uniqueness problem for the representation of a tensor as a sum of terms corresponding to points and tangent vectors of the Segre variety associated with the format of the tensor. We give complete results for unions of one point and one tangent vector.
\end{abstract}

Keywords: Terracini's lemma; secant varieties; zero-dimensional schemes; veronese variety; segre variety

\section{Introduction}

Let $X \subset \mathbb{P}^{r}$ be an integral and non-degenerate $n$-dimensional variety.

To recall the classical notion of abstract secant variety, its map to $\mathbb{P}^{r}$ and its differential (computed in geometric term by A. Terracini) we use the following notation.

For any closed subscheme $Z \subset \mathbb{P}^{r}$ let $\langle Z\rangle$ denote its linear span. Let $X_{\text {reg }}$ denote the set of all smooth points of $X$. For any $o \in X_{\text {reg let }}(2 o, X)$ (or just $2 o$ ) denote the closed subscheme of $X$ with $\left(\mathcal{I}_{0}\right)^{2}$ as its ideal sheaf. The scheme 20 is a zero-dimensional scheme $(20)_{\text {red }}=\{o\}$ and $\operatorname{deg}(2 o)=n+1$. Moreover, $\langle 2 o\rangle$ is the Zariski tangent space of $X$ at $o$. For all finite subsets $S \subset X_{\text {reg }}$ set $2 S:=\cup_{o \in S} 20$.

Fix a positive integer $s<r$. Let $S\left(X_{\text {reg, }} s\right)^{\prime}$ denote the set of all $S \subset X_{\text {reg }}$ such that $\# S=s$ and $S$ is linearly independent. The set $S\left(X_{\text {reg, }} s\right)^{\prime}$ is a smooth quasi-projective variety and its closure $\mathbb{H}(X, s)$ in the Hilbert scheme of $X$ is an integral projective variety, singular if $n>2$. Let $\Sigma_{s}^{0}\left(X_{\text {reg }}\right)$ denote the set of all pairs $(S, q) \in S\left(X_{\text {reg }}, s\right)^{\prime} \times \mathbb{P}^{r}$ such that $q \in\langle S\rangle$ and $q \notin\left\langle S^{\prime}\right\rangle$ for all $S^{\prime} \subsetneq S$. The set $\Sigma_{S}^{0}\left(X_{\text {reg }}\right)$ is a smooth quasi-projective variety. The closure $\Sigma_{s}(X)$ of $\Sigma_{s}^{0}\left(X_{\text {reg }}\right)$ in $\mathbb{H}(X, s) \times \mathbb{P}^{r}$ is a closed and irreducible projective variety, often called the abstract $s$-secant variety of $X$. Call $\tilde{\pi}: \Sigma_{s}(X) \rightarrow \mathbb{P}^{r}$ the morphism induced by the projection $\mathbb{H}(X, s) \times \mathbb{P}^{r} \rightarrow \mathbb{P}^{r}$. The irreducible variety $\sigma_{s}(X):=\tilde{\pi}\left(\Sigma_{s}(X)\right)$ is the $s$-secant variety of $X$, i.e., the closure in $\mathbb{P}^{r}$ of the union of all linear spaces $\langle S\rangle$ for some subset of $X$ with cardinality $s$. However, since $\Sigma_{S}(X)$ is usually very singular (even for nice $X)$ we consider the differential of $\tilde{\pi}$ only at the points of $\Sigma_{s}^{0}\left(X_{\text {reg }}\right)$. Set $\pi:=\tilde{\pi}_{\mid \Sigma_{s}^{0}\left(X_{\text {reg }}\right)}$. Fix $(S, q) \in \Sigma_{S}^{0}\left(X_{\text {reg }}\right)$. A. Terracini proved that the image of the differential $d \pi$ of $\pi$ at $(S, q)$ does not depend on $q$ : it is the linear span of all tangent spaces $T_{o} X, o \in S$, i.e., it is $\langle 2 S\rangle$ ([1], Cor. 1.11). Since Alessandro Terracini's classical papers the study of the differential of $\pi$ gave (for very good reasons: the results were both nice and useful) several papers, most of them for the case $S$ general in $X$ so that the rank of $d \pi$ is the integer $\operatorname{dim} \sigma_{S}(X)$. Some recent papers also considered the case in which $S$ is not general $([2,3])$. Here we consider both cases, $S$ general and $S$ very specific. An arrow of $X_{\text {reg }}$ is a connected degree 2 scheme $v \subset X_{\text {reg. Since } v}$ is assumed to be connected, its reduction $v_{\text {red }}$ is a point,

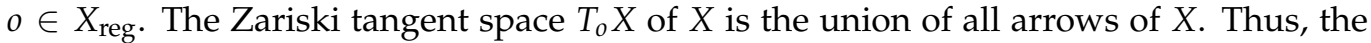
linear span $\langle 2 S\rangle$ has the expected dimension if and only if all unions of $s$ arrows, each of them with as its support a different point of $S$, are linearly independent. This observation due to K. Chandler ([4-6]) had many applications ([6-8]). A union of points and arrows may also be used to describe tensors and homogeneous polynomials. For instance in the 
additive decomposition of degree $d$ forms an arrow corresponds to a term in the sum of the form $\ell^{d-1} \mu$, where $\ell$ and $\mu$ are non-proportional linear forms, while a point corresponds to an addendum $\ell^{d}, \ell \neq 0$.

We consider a general $S$ for the Veronese embeddings $X_{n, d}$ of $\mathbb{P}^{n}$, but we ask if $\langle 2 S\rangle \cap X_{n, d}$ is scheme-theoretically the scheme $2 S$ or not. This is tricky and we discuss in more details why it is tricky in Section 4. For all positive integers $n$ and $d$ let $t(n, d)$ (resp. $t_{1}(n, d)$ ) be the maximal integer $x>0$ such that $h^{1}\left(\mathcal{I}_{Z}(d)\right)=0$ and $\mathcal{I}_{Z}(d)$ is globally generated (resp. $h^{1}\left(\mathcal{I}_{Z}(d)\right)=0$ and $\mathcal{I}_{Z}(d)$ has no base points outside $Z_{\text {red }}$ ), where $Z$ is a general union of $x$ double points of $\mathbb{P}^{n}$. These integers may be expressed with the geometric language used for the additive decomposition of degree $d$ forms in the following way. Let $v_{d}: \mathbb{P}^{n} \rightarrow \mathbb{P}^{r}, r=\left(\begin{array}{c}n+d \\ n\end{array}\right)-1$, denote the order $d$ Veronese embedding of $\mathbb{P}^{n}$, i.e., the embedding given evaluating all degree $d$ forms in $n+1$ variables. Set $X_{n, d}:=v_{d}\left(\mathbb{P}^{n}\right)$. The integer $t(n, s)$ is the maximal integer $x>0$ such that $\operatorname{dim}\langle 2 S\rangle=x(n+1)-1$ and $X_{n, d} \cap\langle 2 S\rangle=2 S$ scheme-theoretically.

We prove the following result.

Theorem 1. Fix integers $d \geq 5$ and $z$ such that $0 \leq 3 z \leq\left(\begin{array}{c}d+2 \\ 2\end{array}\right)-5$. Let $E \subset \mathbb{P}^{2}$ be a general union of $z$ double points. Then $h^{1}\left(\mathcal{I}_{E}(d)\right)=0$ and $\mathcal{I}_{E}(d)$ is globally generated.

The vanishing of $h^{1}\left(\mathcal{I}_{E}(d)\right)$ is well-known, but it is put in the statement because for a non-general $E$ (call if $F$ ) it is easy to obtain $\mathcal{I}_{F}(d)$ globally generated for some $F$ such that $h^{0}\left(\mathcal{I}_{F}(d)\right)>h^{0}\left(\mathcal{I}_{E}(d)\right)$. To prove Theorem 1 we use a degeneration of several planar double points ([9]).

A natural question is the extension of Theorem 2 to the case $n>2$. We ask also for the proof of similar results for tensors of certain formats and certain tensor ranks and for the case of partially symmetric tensors.

In Section 5 we consider the following uniqueness problem for tensors. For which formats there is a concise tensor which can be irredundantly determined by the union of a point and an arrow for more than one union of a point and an arrow? Propositions 1 and 2 and Theorem 3 give the list of the exceptional cases.

The last section is speculative. Suppose that at a certain $(S, q)$ the differential of the Terracini map has a kernel. Is there a condition (using higher derivatives) which says if the fiber of $\pi$ at $(S, q)$ is positive-dimensional?

Unless otherwise stated we work over an algebraically closed field $\mathbb{K}$ of characteristic 0 .

\section{Preliminaries}

Let $X$ be an integral projective variety. Let $D \subset X$ be an effective Cartier divisor of $X$ and $Z$ a zero-dimensional closed subscheme of $X$. The residual scheme $\operatorname{Res}_{D}(Z)$ of $Z$ is the closed subscheme of $X$ with $\mathcal{I}_{Z}: \mathcal{I}_{D}$ as its ideal sheaf. We have $\operatorname{deg}(Z)=\operatorname{deg}(Z \cap D)+$ $\operatorname{deg}\left(\operatorname{Res}_{D}(Z)\right)$. For every line bundle $\mathcal{L}$ on $X$ the following sequence

$$
0 \rightarrow \mathcal{I}_{\operatorname{Res}_{D}(Z)} \otimes \mathcal{L}(-\mathcal{D}) \rightarrow \mathcal{I}_{\mathcal{Z}} \otimes \mathcal{L} \rightarrow \mathcal{I}_{\mathcal{Z} \cap \mathcal{D}, \mathcal{D}} \otimes \mathcal{L}_{\mid \mathcal{D}} \rightarrow \prime
$$

is exact. We will say that (1) is the residual exact sequence of $D$ (without mentioning $Z$ and $\mathcal{L})$. Take $o \in X_{\text {reg }} \cap D_{\text {reg. We have }}(2 o, X) \cap D=(2 o, D)$ and $\operatorname{Res}_{D}((2 o, X))=\{o\}$. Let $v \subset X$ be an arrow such that $v_{\text {red }}=\{o\}$. If $v \subset D$, then $\operatorname{Res}_{D}(v)=\varnothing$. If $v \nsubseteq D$, then $V \cap D=\{o\}$ (as schemes) and $\operatorname{Res}_{D}(v)=\{o\}$. If $H \subset X$ is an effective Cartier divisor, then $\operatorname{Res}_{H}\left(\operatorname{Res}_{D}(Z)\right)=\operatorname{Res}_{H+D}(Z)$, where $H+D$ denote the sum as effective divisors.

Let $X \subset \mathbb{P}^{r}$ be an integral and non-degenerate variety. For any $q \in \mathbb{P}^{r}$ the $X$-rank $r_{X}(q)$ of $q$ is the minimal cardinality of a finite subset of $X$ whose linear span contains $q$.

Fix a quasi-projective variety $T$ of dimension at least 2 and $o \in T_{\text {reg. }}$. Let $v, w \subset T$ be arrows such that $v \neq w$ and $v_{\text {red }}=w_{\text {reg }}=\{o\}$. The scheme $v \cup w$ is called a planar double point or a planar double point of $T$. The next remark explains the properties of planar double points. 
Remark 1. Fix a quasi-projective variety $T$ of dimension at least 2 and $o \in T_{\text {reg. }}$ Let $v, w \subset T$ be arrows such that $v \neq w$ and $v_{\text {red }}=w_{\text {reg }}=\{o\}$. Set $Z:=v \cup w$. We have $\operatorname{deg}(Z)=3$ and $Z_{\text {red }}=1$. There are $\infty^{1}$ arrows $z \subset T$ such that $z_{\text {red }}=\{o\}$ and $z \subset Z$. Moreover, if $z \neq v$, then $Z=v \cup z$. The Zariski tangent space of $Z$ at $o$ has dimension 2 and there is a quasi-projective variety $M \subseteq T$ such that $\operatorname{dim} M=2, o \in M_{\text {reg }}$ and $Z \subset M$. If $\operatorname{dim} T=2$, then we take $M=T$, but if $\operatorname{dim} T>2$ there are infinitely many quasi-projective varieties $M \subseteq T$ such that $\operatorname{dim} M=2$, $o \in M_{\text {reg }}$ and $Z \subset M$. Fix any such $M$. The scheme $Z$ is the closed subscheme of $M$ with $\left(\mathcal{I}_{o, M}\right)^{2}$ as its ideal sheaf. If $T$ is embedded in a projective space, then $Z$ spans a plane.

\section{Veronese Varieties}

In this section, we prove Theorem 2. In Section 4 we discuss why it does not follow from known results on weak nondefectivity and tangenential nondefectivity ([10-13]).

Obviously $t(1, d)=t_{1}(n, d)=\lceil d / 2\rceil$ for all $d \geq 1$.

For all positive integers $n$ and $d$ let $\alpha(n, d)$ denote the maximal cardinality of a finite set $S \subset \mathbb{P}^{n}$ such that $h^{1}\left(\mathcal{I}_{2 S}(d)\right)=0$. Obviously $\alpha(1, d)=\lceil d / 2\rceil$ and $\alpha(n, 1)=1$. Since the singular locus of a quadric hypersurface is a linear space, $\alpha(n, 2)=1$. A key theorem due to Alexander and Hirschowitz ([14-17]) computes $\alpha(n, d)$ for all $n, d$ and says that $\alpha(n, d)=\left\lfloor\left(\begin{array}{c}n+d \\ n\end{array}\right) /(n+1)\right\rfloor$ for all $d \geq 3$, unless $(n, d) \in\{(2,4),(3,4),(4,3),(4,4)\}$. Moreover, $\alpha(2,4)=4, \alpha(3,4)=8, \alpha(4,3)=6$ and $\alpha(4,4)=13$.

Remark 2. Obviously $t(n, d) \leq t_{1}(n, d) \leq \alpha(n, d)$. Please note that $t_{1}(n, d) \leq \alpha(n, d)-1$ if $n \geq 2$ (and hence $\mathcal{I}_{2 S}(d)$ is not the trivial line bundle) and $\left(\begin{array}{c}n+d \\ n\end{array}\right)-(n+1) \alpha(n, d) \leq n$ for all $n \geq 2$ and $d \geq 3$, except in the 4 exceptional cases $(n, d) \in\{(2,4),(3,4),(4,3),(4,4)\}$.

Remark 3. Let $S \subset \mathbb{P}^{n}$ be a general subset such that $\# S=\alpha(n, d-1)$. The Castelnuovo-Mumford lemma gives that $\mathcal{I}_{2 S}(d)$ is spanned and that $h^{1}\left(\mathcal{I}_{2 S}(d)\right)=0$. Thus, $t(n, d) \geq \alpha(n, d-1)$.

Remark 4. Since any 2 points of a projective space are collinear, $t(n, 3)=t_{1}(n, 3)=1$. Take $n=2$ and $d=4$. Since 4 general points of $\mathbb{P}^{2}$ are the complete intersection of 2 conics and $\alpha(2,4)=4$, we have $t(2,4)=t_{1}(2,4)=4$. Thus, in a few cases the upper bound in Remark 3 is sharp.

Lemma 1. $t(2,5)=t_{1}(2,5)=6$.

Proof. Since $\alpha(2,5)=7$ and $3 \cdot 7=\left(\begin{array}{l}7 \\ 2\end{array}\right), t_{1}(2,5) \leq 6$. Thus, it is sufficient to prove that $t(2,5) \geq 6$. Fix a smooth cubic $D \subset \mathbb{P}^{2}$ and take $S_{1} \subset D$ such that $\# S_{1}=5$. Take $o \in \mathbb{P}^{2} \backslash D$ such that $o$ is not contained in any line spanned by 2 of the points of $S_{1}$. We need to prove that $\mathcal{I}_{2 S}(5)$ has no base points outside $S$ and that $h^{1}\left(\mathcal{I}_{2 S}(5)\right)=0$. We have $\operatorname{Res}_{D}(2 S)=2 o \cup S_{1}$. Since $\mathcal{O}_{\mathbb{P}^{2}}(1)$ is very ample, the residual exact sequence of $D$ shows that $\mathcal{I}_{S_{1} \cup 2 o}(3)$ has no base locus outside $D \cup\{o\}$. Thus, it is sufficient to use that $D \cong \mathbb{P}^{1}$ is projectively normal and that $\mathcal{O}_{D}(5)\left(-2\left(2 S_{1}, D\right)\right) \cong \mathcal{O}_{D}$.

To prove Theorem 1 we prove the following result.

Theorem 2. Fix integers $d \geq 5$ and $z$ such that $0 \leq 3 z \leq\left(\begin{array}{c}d+2 \\ 2\end{array}\right)-4$. Then there is a connected zero-dimensional scheme $Z \subset \mathbb{P}^{2}$ such that $Z$ is a flat limit of a family of $z$ pairwise disjoint double points, $h^{1}\left(\mathcal{I}_{Z}(d)\right)=0$ and $\left|\mathcal{I}_{Z}(d)\right|$ has no base points outside $Z_{\text {red }}$.

Lemma 2. Let $C \subset \mathbb{P}^{2}$ be a smooth curve. Fix $o \in C$ and positive integers $z$, $w$ such that $3 z / 2<w \leq 2 z$. Then there is a zero-dimensional scheme $Z \subset \mathbb{P}^{2}$ such that $Z_{\mathrm{red}}=\{o\}$, $\operatorname{deg}(Z)=3 z, Z$ is a flat limit of a family of unions of $z$ pairwise disjoint double points, $Z \subset 2 C$ and $\operatorname{deg}(Z \cap C)=w$.

Proof. If $w=2 z$ we use ([18], Proposition 5.1.2), which corresponds in the set-up of [9] to the front collision, which just adds the escaliers of the $z$ double points. As- 
sume $3 z / 2<w<2 z$ and set $e:=2 z-w$. Please note that $w=2(z-e)+e$. We take a specialization $Z$ of a family of general unions $A=B \cup E$ with $B$ a general unions of $z-e$ double points of $\mathbb{P}^{2}$ with as reduction general points of $C$ and $E$ a general union of $e$ double points of $\mathbb{P}^{2}$. Then we apply $e$ times (each time to a different connected component of $E$ ) ([18], §5.2) with the escalier $(2,1)$ of a double point of $\mathbb{P}^{2}$.

Proof of Theorem 2: Let $t$ be the maximal positive integer such that $t \leq d$ and $\left(\begin{array}{c}d+2 \\ 2\end{array}\right)-$ $\left(\begin{array}{c}d-t+2 \\ 2\end{array}\right)-2 \leq 2 z$.

Claim 1: $t>d / 2$.

Proof of Claim 1: Assume $t \leq d / 2$. Since $t$ is maximal, $\left(\begin{array}{c}d+2 \\ 2\end{array}\right)-\left(\begin{array}{c}d / 2+1 \\ 2\end{array}\right) \leq 2 z-1$, i.e., $\left(3 d^{2}+\right.$ $10 d+4) / 8 \leq 2 z-1$, i.e., $16 z \geq 3 d^{2}+10 d+12$. We have $3 z \leq\left(\begin{array}{c}d+2 \\ 2\end{array}\right)-4$, i.e., $6 z \leq$ $d^{2}+3 d-6$, contradicting the assumption $d \geq 5$.

Fix a general $C \in\left|\mathcal{O}_{\mathbb{P}^{2}}(t)\right|$. Thus, $C$ is smooth and $h^{0}\left(\mathcal{O}_{C}(d)\right)=\left(\begin{array}{c}d+2 \\ 2\end{array}\right)-\left(\begin{array}{c}d-t+2 \\ 2\end{array}\right)$. Set $w:=\left(\begin{array}{c}d+2 \\ 2\end{array}\right)-\left(\begin{array}{c}d-t+2 \\ 2\end{array}\right)-2$. By Lemma 2 there is a connected zero-dimensional scheme $Z \subset \mathbb{P}^{2}$ such that $Z \subset 2 C$ and $\operatorname{deg}(Z \cap C)=w$. Thus, $\operatorname{Res}_{C}(Z)$ is a general connected zero-dimensional subscheme of $C$ of degree $3 z-w$. By [19] $h^{1}\left(C, \mathcal{I}_{Z \cap C, C}(d)\right)=0$ and $h^{1}\left(C, \mathcal{I}_{\operatorname{Res}_{C}(Z)}(t)\right)=0$. Since $h^{1}\left(C, \mathcal{I}_{\operatorname{Res}_{C}(Z)}(t)\right)=0$ and $C$ is projectively normal, $h^{1}\left(\mathcal{I}_{\operatorname{Res}_{C}(Z)}(t)\right)=0$. The residual exact sequence of $C$ gives $h^{1}\left(\mathcal{I}_{Z}(d)\right)=0$ and that the restriction map $\rho: H^{0}\left(\mathcal{I}_{Z}(d)\right) \rightarrow H^{0}\left(C, \mathcal{I}_{C \cap Z, C}(d)\right)$ is surjective. Call $W$ the scheme-theoretic base locus of $\left|\mathcal{I}_{Z}(d)\right|$. Since $C$ is a smooth curve and $Z \cap C$ is a general connected degree $w$ subscheme of $C$, [20] gives that no point of $C \backslash C \cap Z$ is a base point of $H^{0}\left(C, \mathcal{I}_{C \cap Z, C}(d)\right)$. The surjectivity of $\rho$ gives that no point of $C \backslash Z_{\text {red }}$ is a base point of $H^{0}\left(\mathcal{I}_{Z}(d)\right)$. Since a general osculating space of a curve is not hyperosculating and $\rho$ is surjective, we obtain $C \cap W=C \cap Z$ as schemes. Since $t>d / 2, H^{0}\left(\mathcal{O}_{\mathbb{P} \in}(\lceil-\sqcup)) \cong \mathcal{H}^{\prime}\left(\mathcal{O}_{\mathcal{C}}(\lceil-\sqcup))\right.\right.$. Since $\operatorname{Res}_{C}(Z)$ is a general connected degree $3 z-w$ subscheme of the smooth curve $C$ and $h^{0}\left(\mathcal{O}_{\mathcal{C}}(\lceil-\sqcup)) \geq \ni \ddagger-\sqsupseteq+\in,[20]\right.$ gives $W \cap 2 C=Z$. Thus, $\mathcal{I}_{Z}(t)$ has no base point outside $C$. Since no point of $C \backslash Z_{\text {red }}$ is a base point of $H^{0}\left(\mathcal{I}_{Z}(d)\right)$, no $a \in \mathbb{P}^{2} \backslash Z_{\text {red }}$ is a base point of $\left|\mathcal{I}_{Z}(d)\right|$.

Proof of Theorem 1: Being globally generated is an open condition in families of coherent sheaves with constant cohomology. Thus, it is sufficient to prove that if $3 z \neq\left(\begin{array}{c}d+2 \\ 2\end{array}\right)-4$ the scheme $Z$ constructed in the proof of Theorem 2 is globally generated. Let $\mathcal{I}_{W}(d) \subseteq \mathcal{I}_{Z}(d)$ be the image of the evaluation map $H^{0}\left(\mathcal{I}_{Z}(d)\right) \otimes \mathcal{O}_{\mathbb{P} 2} \rightarrow \mathcal{I}_{Z}(d)$. Since $W \supseteq Z$, to conclude the proof it is sufficient to prove that $W \subseteq Z$. Since $\left|\mathcal{I}_{Z}(d)\right|$ has no base points outside $Z_{\text {red }}, W$ is a connected zero-dimensional scheme. Take $C, t$ and $w$ as in the proof of Theorem 2. We saw that $W \cap C=Z \cap C$. Since $\operatorname{deg}(Z) \leq\left(\begin{array}{c}d+2 \\ 2\end{array}\right)-4, \operatorname{deg}\left(\operatorname{Res}_{C}(Z)\right) \leq$ $h^{0}\left(\mathcal{O}_{\mathcal{C}}(\lceil-\sqcup))-\in\right.$. Please note that $\operatorname{Res}_{C}(Z)$ is a general connected zero-dimensional scheme of degree $3 z-w \leq h^{0}\left(\mathcal{O}_{\mathcal{C}}\left(\lceil-\lceil\lceil/ \in\rceil))-\in\right.\right.$. Hence $H^{0}\left(C, \mathcal{I}_{\operatorname{Res}_{C}(Z)}(d-\lceil d / 2\rceil)\right)$ has no base points ([20]; note that in [20] "curve" means "smooth curve"). Consider the residual exact sequence

$$
0 \rightarrow \mathcal{I}_{\operatorname{Res}_{C}(Z)}(d-t) \rightarrow \mathcal{I}_{Z}(d) \rightarrow \mathcal{I}_{Z \cap C, C}(d) \rightarrow 0
$$

We saw that $h^{1}\left(\mathcal{I}_{\operatorname{Res}_{C}(Z)}(d-t)\right)=h^{1}\left(C, \mathcal{I}_{Z \cap C, C}(d)\right)=0$ and that $\mathcal{I}_{\operatorname{Res}_{C}(Z)}(d-t)$ and $\mathcal{I}_{Z \cap C, C}(d)$ are globally generated. Thus, $\mathcal{I}_{Z}(d)$ is globally generated.

\section{Base Point Freeness}

In this section, we point out why it is still open, even for generic symmetric rank, although very similar statements are true, stated and proved in the literature ([10-13]).

Assume characteristic zero.

Let $X \subset \mathbb{P}^{r}$ be an integral and non-degenerate variety. Set $n:=\operatorname{dim} X$ and fix an integer $s>0$ such that $s(n+1) \leq r$ and $\operatorname{dim} \sigma_{s}(X)=s(n+1)-1$, i.e., $X$ is not secant 
$s$-defectivity. Fix a general $S \subset X_{\text {reg }}$ such that $\# S=s$ and set $Z:=\cup_{o \in S} 2 o$. Since $\operatorname{dim} \sigma_{s}(X)=s(n+1)-1, \mathbb{L}:=\langle Z\rangle$ has dimension $s(n+1)-1$. Take a general hyperplane $H \subset \mathbb{P}^{r}$ containing $Z$. There are key notions due to C. Ciliberto and L. Chiantini (weak nondefectivity and tangential nondefectivity) ([10-12]) which when they are satisfies imply that $H$ is tangent to $X_{\text {reg }}$ only at the points of $S$. This true statement does not imply that $\mathbb{L}$ meets $X_{\text {reg }}$ only at $S$ and the linear spaces contained in $X$ and containing at least one point of $S$, even when $\mathbb{L}$ has codimension $\geq n+1$. It would seem intuitively true, but it is only conjectural for curves and false in some cases for higher-dimensional smooth manifolds ([21]).

We say that Assumption 1 holds if the following conjectural statement is true:

Assumption 1. Assume char $(\mathbb{K})=0$. Let $X \subset \mathbb{P}^{r}, r \geq 3$, be any integral and non-degenerate curve. For a general $p \in X_{\text {reg }}$ the tangent line $T_{p} X$ of $X$ at $p$ meets $X$ only at $p$.

An assumption similar to Assumption 1 trivially fail for all $X$ such that $\operatorname{dim} X>1$ and $X$ is covered by lines. However, an example due to M. Ohno shows that it may fail even for smooth manifolds of general type [21]. See [22,23] for many partial solutions and applications of Assumption 1. Thus, we cannot freely extend to general unions of double points the following observation concerning general finite sets.

Remark 5. Let $X \subsetneq \mathbb{P}^{r}$ be an integral and non-degenerate variety. Set $n:=\operatorname{dim} X$. Let $S \subset X$ be a general set such that $\# S=r-n$. In characteristic 0 an easy application of the linear general position of a general codimension $n$ linear section gives $S=X \cap\langle S\rangle$ (scheme-theoretic intersection).

For any $o \in X_{\text {reg }}$ and all positive integer $m$ let mo denote the closed subscheme of $X_{\text {reg }}$ with $\left(\mathcal{I}_{0}\right)^{m}$ as its ideal sheaf. The linear space $O_{m}(X)_{o}:=\langle(m+1) o\rangle$ is the $m$ osculating linear space of $X$ at $o$. Motivated by [20] we consider the following Condition (Assumption 2):

Assumption 2. Assume char $(\mathbb{K})=0$. Fix integers $r \geq m+2 \geq 3$. Let $X \subset \mathbb{P}^{r}$ be any integral and non-degenerate curve. For a general $p \in X_{\text {reg }}$ the $m$ osculating space $O_{m}(X)_{p}$ of $X$ at $p$ meets $X$ only at $p$ and the support of the union of the point and the arrow. Usually, this support is a very small part of the Segre variety.

We are working over an algebraically closed field of characteristic zero, because Assumption 1 fails in positive characteristic even for some smooth curves ([24], Example 4.1). In characteristic 0 Assumptions 1 and 2 holds for all smooth curves ([20,23,24], Theorem 3.1)

\section{Tensors}

Tensors associated with an arrow are exactly the tensors contained in the tangential variety of the Segre variety related to the format of the tensor. In this section we describe all concise tensors which are linear combinations in two different ways of a rank 1 tensor and a tensor associated with an arrow (Theorem 3). Theorem 3 lists 7 cases with for each case a quotation of an example or remark of the paper. The remark or example describes in detail each exceptional case. In each case we describe by how many parameters the possible unions of an arrow and a point depend.

We recall the following properties of the Segre varieties and their connection with tensors and the tensor rank of a tensor ([25]).

Let $Y=\mathbb{P}^{n_{1}} \times \cdots \times \mathbb{P}^{n_{k}}, k \geq 1, n_{i}>0,1 \leq i \leq k$, be a multiprojective space. Let $\pi_{i}: Y \rightarrow \mathbb{P}^{n_{i}}$ denote the projection of $Y$ onto its $i$-th factor. If $k \geq 2$ set $Y_{i}:=\prod_{h \neq i} \mathbb{P}^{n_{h}}$ and let $\eta_{i}: Y \rightarrow Y_{i}$ denote the projection. The map $\eta_{i}$ is the map forgetting the $i$-th coordinate of each $\left(a_{1}, \ldots, a_{k}\right) \in Y$. Let $v_{i}$ denote the Segre embedding of $Y_{i}$.

For all $\left(d_{1}, \ldots, d_{k}\right) \in \mathbb{Z}^{k}$ set $\mathcal{O}_{Y}\left(d_{1}, \ldots, d_{k}\right):=\otimes_{i=1}^{k} \pi_{i}^{*}\left(\mathcal{O}_{\mathbb{P}^{n_{i}}}\left(d_{i}\right)\right)$. The line bundles $\mathcal{O}_{Y}\left(d_{1}, \ldots, d_{k}\right),\left(d_{1}, \ldots, d_{k}\right) \in \mathbb{Z}^{k}$, form a $\mathbb{Z}$-basis of the abelian group Pic $(Y)$. The Künneth formula gives $h^{0}\left(\mathcal{O}_{Y}\left(d_{1}, \ldots, d_{k}\right)\right)=0$ if some $d_{i}<0, h^{0}\left(\mathcal{O}_{Y}\left(d_{1}, \ldots, d_{k}\right)\right)=\prod_{i=1}^{k}\left(\begin{array}{c}n_{i}+d_{i} \\ n_{i}\end{array}\right)$ if 
$d_{i} \geq 0$ for all $i$ and $h^{1}\left(\mathcal{O}_{Y}\left(d_{1}, \ldots, d_{k}\right)\right)=0$ if $d_{i} \geq-1$ for all $i$. For any $i \in\{1, \ldots, k\}$ let $\mathcal{O}_{\mathcal{Y}}(\uparrow)\left(\right.$ resp. $\left.\mathcal{O}_{Y}\left(\hat{\varepsilon}_{i}\right)\right)$ be the line bundle $\mathcal{O}_{\mathcal{Y}}\left(\dashv_{\infty}, \ldots, \dashv_{\|}\right)$on $Y$ with multidegree $\left(a_{1}, \ldots, a_{k}\right)$ with $a_{i}=1$ and $a_{j}=0$ for all $j \neq i$ (resp. We have $\left.h^{0}\left(\mathcal{O}_{\mathcal{Y}}(\uparrow)\right)=\backslash\right\rangle+\infty$. Set $r:=$ $-1+\prod_{i=1}^{k}\left(n_{i}+1\right)$. Let $v: Y \rightarrow \mathbb{P}^{r}$ denote the Segre embedding of $Y$.

For any tensor $T$ of format $\left(n_{1}+1\right) \times \cdots \times\left(n_{k}+1\right), T \neq 0$, the tensor rank of $T$ is the $v(Y)$-rank $r_{v(Y)}([T])$ of the element $[T] \in \mathbb{P}^{r}$ associated with $T$.

The main result of this section is the following one.

Theorem 3. Let $A \subset Y=\mathbb{P}^{n_{1}} \times \cdots \times \mathbb{P}^{n_{k}}$ be a union of a point and an arrow. Assume that $Y$ is the minimal multiprojective space containing $Y$. Fix $q \in\langle v(A)\rangle$ such that $\left.q \notin v\left(A^{\prime}\right)\right\rangle$ for any $A^{\prime} \subseteq A$. Assume the existence of a union $B \subset Y$ of a point and an arrow such that $B \neq A$ and $q \in\langle v(A)\rangle \cap\langle v(B)\rangle$. Then $Y$ is as in one of the following cases (assuming $n_{i} \geq n_{j}$ for all $i \leq j$ ):

1. $\operatorname{deg}(B)=1$ and either $k=1, n_{1} \leq 2$ or $k=2$ and $n_{1}=n_{2}=1$.

2. $\operatorname{deg}(B)=2$ and either $k=1, n_{1} \leq 2$ or $k=2$ and $n_{1}=n_{2}=1$.

3. $k=2, n_{1} \leq \operatorname{deg}(B)-1$ and $A, B, q$ are as in Remark 10 .

4. $\operatorname{deg}(B)=3, Y=\left(\mathbb{P}^{1}\right)^{3}$ and $A, B, q$ are as in Remark 11 .

5. $\operatorname{deg}(B)=3, Y=\left(\mathbb{P}^{1}\right)^{4}$ and $A, B, q$ are as in Example 9.

6. $\operatorname{deg}(B)=3, Y=\mathbb{P}^{2} \times \mathbb{P}^{1} \times \mathbb{P}^{1}$ and $A, B$ are as in Example 3 .

7. $\operatorname{deg}(B)=3$ and $(Y, q, B)$ is as in Examples $4,5,6,7$ or 8 .

In each case we give a rough description of the possible $B^{\prime}$ s. The one with $\operatorname{deg}(B)=1$ (resp. $\operatorname{deg}(B)=2$ ) are listed in Remark 10 (resp. 11). The last case, i.e., Examples 4, 5, 7 or 8 are the only cases which allow any $k \geq 5$.

Remark 6. Let $Z \subset Y$ be a zero-dimensional scheme of degree $z \geq 2$. The minimal multiprojective subspace of $Y$ containing $Z$ is the multiprojective space $Y^{\prime}:=\prod_{i=1}^{k}\left\langle\pi_{i}(Z)\right\rangle$. Write $Y^{\prime}=\mathbb{P}^{m_{1}} \times$ $\cdots \times \mathbb{P}^{m_{s}}$ for some positive integers $s$ and $m_{h}, 1 \leq h \leq s$. We have $m_{i} \leq \operatorname{deg}(Z)-1$ for all $i$ and this is in general the only restriction we may obtain from the isomorphism class of $Z$ as an abstract scheme. Of course, $s \leq k$ and if the $h$-th positive-dimensional factor of $Y^{\prime}$ is contained in the $i$-th-dimensional factor of $Y$, then $m_{h} \leq n_{i}$.

Remark 7. ([26], Lemma 4.4) Let $W \subset Y$ be a zero-dimensional scheme such that $\operatorname{deg}(W) \leq 3$ and $v(W)$ is linearly dependent. Since $v$ is an embedding, $\operatorname{deg}(W)=3$ and $\langle v(W)\rangle$ is a line. Since $v(Y)$ is scheme-theoretically cut out by quadrics and $W \subseteq\langle v(E)\rangle \cap v(Y)$, then $\langle v(E)\rangle \subset Y$. Since the only linear subspaces contained in the Segre variety $X$ are the one contained in a fiber of one of its $k$ rulings, there is of $i \in\{1, \ldots, k\}$ such that $\operatorname{deg}\left(\pi_{h}(W)\right)=1$ for all $h \neq i, \pi_{i \mid W}$ is an embedding and $\pi_{i}(W) \subseteq \mathbb{P}^{n_{i}}$ is a line.

Remark 8. Take $q \in\langle v(B)\rangle$ with $B \subset Y, \operatorname{deg}(B) \leq 3$ and $B$ curvilinear. The point $q$ has border rank $b \leq \operatorname{deg}(A)$ ([27], Proposition 1.1 and Theorem 1.2) and the minimal multiprojective space $Y^{\prime} \subseteq Y$ with $q \in\left\langle v\left(Y^{\prime}\right)\right\rangle$ contains all curvilinear degree $b$ schemes $E \subset Y$ such that $\operatorname{deg}(E)=b$ and $q \in\langle v(E)\rangle$ are contained in $Y^{\prime}([28]$, Theorem 2.5).

Example 1. Let $W \subset \mathbb{P}^{n}$ be a zero-dimensional scheme such that $\operatorname{deg}(W)=4, W$ spans $\mathbb{P}^{n}, W$ is linearly dependent, but all proper subschemes are linearly independent. Obviously $n=2$. Since $h^{0}\left(\mathcal{O}_{\mathbb{P}^{4}}(2)\right)=6$ and $\operatorname{deg}(L \cap W) \leq 3$ for all lines $L \subset \mathbb{P}^{2}, \operatorname{dim}\left|\mathcal{I}_{W}(2)\right|=2$ and either $W$ is the complete intersection of 2 conics or $\operatorname{deg}(L \cap W)=3$ for exactly one line $L$ and there is $o \in \mathbb{P}^{2}$ such that all $E \in\left|\mathcal{I}_{W}(2)\right|$ is of the form $E=L \cup R$ with $R \in\left|\mathcal{I}_{o}(1)\right|$. In both cases there are non-curvilinear $W$ 's. In the latter case the latter case $o \in L$ and $W$ is the union of the fat point 20 and some $p \in L \backslash\{0\}$.

Example 2. Let $W \subset \mathbb{P}^{1} \times \mathbb{P}^{1}$ be a degree 4 scheme. Since $h^{0}\left(\mathcal{O}_{\mathbb{P}^{1} \times \mathbb{P}^{1}}(1,1)\right)=4$, $h^{1}\left(\mathcal{I}_{W}(1,1)\right)>0$ if and only if there is $D \in\left|\mathcal{O}_{\mathbb{P} \infty \times \mathbb{P} \infty}(\infty, \infty)\right|$ containing $W$. If $v\left(W^{\prime}\right)$ is linearly independent for all $W^{\prime} \subsetneq W$, then $D$ is unique, because $h^{1}\left(\mathcal{I}_{W}(1,1)\right)=1$ in this case. 
Proposition 1. Let $v \subset Y$ be an arrow such that $Y$ is the minimal multiprojective space. Set $o:=v_{\text {red. Fix }} q \in\langle v(v)\rangle \backslash\{o\}$. There is a zero-dimensional scheme $Z \subset Y$ such that $\operatorname{deg}(Z) \leq 2$, $Z \neq v$, and $q \in\langle v(Z)\rangle$ if and only if one of the following cases occur:

1. $k=1, n_{1}=1$;

2. $k=1, n_{1}=2$;

3. $k=2, n_{1}=n_{2}=1$.

(a) In case (1) we may take $\operatorname{deg}(Z)=1$ (with $v(Z)=\{q\}), \operatorname{deg}(Z)=2$ and reduced or $\operatorname{deg}(Z)=2$ and $Z$ an arrow.

(b) In case (2) $v(v \cup Z)$ is the complete intersection of two conics in the plane $\langle v(W \cup Z)\rangle$.

(c) In case (3) there are $\infty^{2}$ reduced $Z$ (parametrized by a plane minus a line) and $\infty^{1}$ arrows $Z$ (parametrized by a line minus the point corresponding to $v$ ).

Proof. Cases (1), (2) and (3) occur and in each case the possible schemes Z described in (a), (b) and (c) are the schemes which occur (Examples 1 and 2).

Now we prove the "only if" part. Set $W:=v \cup Z$. Thus, $\operatorname{deg}(W) \leq 4$. Since $v \neq Z$, $\operatorname{deg}(W) \leq 3$. First assume $\operatorname{deg}(W)=3$. We are in the case (1) by Example 1. Now assume $\operatorname{deg}(W)=4$ and hence $Z \cap v=\varnothing$. Theorem 7 gives that we are in one of these cases (1), (2) and (3).

Remark 9. Proposition 1 describes all degree 4 schemes $W \subset Y, W$ not reduced and not containing a connected component of degree at least 3 , such that $Y$ is the minimal multiprojective space containing $W, v(W)$ is linearly dependent and all proper subschemes of $v(W)$ are linearly independent. For the case $W$ reduced, see [29] and/or [30]. For an arbitrary $W$ of degree 4, see [31]. In [26,29-31] there are related results obtained under assumptions with minor differences. For instance in [26] we assume that $q$ has rank 3 and that $Y$ is the minimal multiprojective space such that $q \in\langle v(Y)\rangle$. Here we do not assume that $v(Y)$ is the minimal Segre spanning $q$, because it seems too restrictive. Making the assumption that $v(Y)$ is concise for $q$ would drastically cut some proofs. Requiring that $q$ has not cactus rank $\leq 2$ would allow the interested reader to omit Propositions 1 and 2.

Remark 10. Assume $k=2$.

(a) Assume $n_{i}=1$ for at least one $i$, say $n_{2}=1$. Since any $\left(n_{1}+1\right) \times 2$ matrix has rank $\leq 2$, for each $q \in \mathbb{P}^{r} \backslash Y$ there are infinitely many $S \subset Y$ such that $q \in\langle v(S)\rangle$ and $\# S=2$. Now assume $n_{1} \leq 2$. Since $\mathbb{P}^{r}$ is the tangential variety of $\tau(v(Y))$, we obtain the existence of an arrow $Z \subset Y$ such that $q \in\langle v(Z)\rangle$. By ([32], Ex. II.3.22(b)) we see that $q$ is associated with at least $\infty^{2}$ sets $S$ and $\infty^{1}$ arrows $Z$.

(b) Assume $n_{1}=n_{2}=2$ and hence $r=8$. There are $q \in \mathbb{P}^{8}$ with tensor rank 2 and tensors spanned by $v(Z)$ with $Z$ connected and of degree 2. As in part (a) we obtain $q \in\langle v(A)\rangle$ with $\operatorname{deg}(A)=3$ and $A$ union of an arrow and a point.

Proposition 2. Let $A \subset Y$ be the union of an arrow $v$ and a point $p \neq 0:=v_{\text {red. Assume }}$ that $Y$ is the minimal multiprojective space containing $A$. Fix $q \in\langle v(A)\rangle$ such that there is no $A^{\prime} \subsetneq A$ with $q \in\left\langle v\left(A^{\prime}\right)\right\rangle$. There is a zero-dimensional scheme $Z \subset Y$ such that $\operatorname{deg}(Z) \leq 2$ and $q \in\langle v(Z)\rangle$ if and only if $A, Y$ and $Z$ are as follows:

1. $k=1$ and $n_{1}=1$ (here $q \in v(Y)$, say $q=v\left(o^{\prime}\right)$ );

2. $k=2, n_{1}=n_{2}=1$;

3. $k=2, n_{1}+n_{2}=3 ; \quad k=n_{1}=n_{2}=2$;

4. $k=3, n_{1}=n_{2}=n_{3}=1$.

(i) In case (1) the schemes $Z$ are as follows:

(i1) $\operatorname{deg}(Z)=1$ and $Z$ is the point of $Y$ such that $q=v(Z)$;

(i2) $\quad Z$ is any degree 2 subscheme.

(ii) In case (2) there are $\infty^{2}$ schemes $Z$ formed by 2 points and $\infty^{1}$ schemes $Z$ which are arrows. 
(iii) In cases (3) and (4) there are at least $\infty^{2}$ schemes $Z$ formed by 2 points and at least $\infty^{1}$ schemes $Z$ which are arrows; in this case $Y$ is not the minimal multiprojective space such that $q \in\langle v(Y)\rangle$.

Proof. All listed cases are associated with some $Z$ and the schemes $Z$ are as described in Remarks 10 and 11. Thus, it is sufficient to prove the "only if" part.

Since $\operatorname{deg}(A)=3$ and $Y$ is the minimal multiprojective space containing $A, n_{i} \leq 2$ for all $i$.

Set $W:=Z \cup A$. Please note that $\operatorname{deg}(W) \leq 5$. Since there is no $A^{\prime} \subsetneq A$ such that $q \in\left\langle v\left(A^{\prime}\right)\right\rangle$, we have $\operatorname{deg}(W) \geq 4$. Since $Y$ is the minimal multiprojective space containing $A, n_{i} \leq 2$ for all $i$. If $n_{i}=1$, then $\operatorname{deg}\left(\pi_{i}(A)\right) \geq 2$. If $n_{i}=2$, then $\operatorname{deg}\left(\pi_{i}(A)\right)=3$ and $\pi_{i}(A)$ is linearly independent. Proposition 1 covers the case $\operatorname{deg}(W)=4$. Thus, we may assume $\operatorname{deg}(W)=5$, i.e., $Z \cap A=\varnothing$.

If $k=1$, then $Z$ is any degree 2 scheme spanning a line containing $o^{\prime}$ and not intersecting $A$. All cases with $k=2$ are covered by Remark 10 . Thus, we may assume $k>2$.

(a) Assume for the moment $n_{i}>1$ for some $i$, say $n_{1}>1$ and hence $n_{1}=2$. Since $Y$ is the minimal multiprojective space containing $Y, \operatorname{deg}\left(\pi_{1}(A)\right)=3$ and $\pi_{1}(A)$ is linearly independent. Take $H_{1} \in\left|\mathcal{I}_{v}\left(\varepsilon_{1}\right)\right|$ and $H_{2} \in\left|\mathcal{I}_{u}\left(\varepsilon_{2}\right)\right|$. The scheme $E:=\operatorname{Res}_{H_{1} \cup H_{2}}(W)$ is contained in $Z$.

(a1) Assume $E \neq \varnothing$. Since $Z \cap A=\varnothing$, quoting ([33], Lemma 5.1) we obtain $h^{1}\left(\mathcal{I}_{E}(0,0,1, \ldots, 1)\right)=0$. Since $E \subseteq Z$ and $\mathcal{O}_{Y}(0,0,1, \ldots, 1)$ is globally generated, we obtain $E=Z$ and $\operatorname{deg}\left(\pi_{i}(Z)\right)=1$ for all $i>2$. Thus, $\left|\mathcal{I}_{Z}\left(\varepsilon_{3}\right)\right| \neq \varnothing$. Fix $M \in\left|\mathcal{I}_{Z}\left(\varepsilon_{3}\right)\right|$. Since $Z \cap A=\varnothing$ and $A \nsubseteq M$, ([33], Lemma 5.1) gives $h^{1}\left(\mathcal{I}_{\operatorname{Res}_{M}(W)}\left(\hat{\varepsilon}_{3}\right)\right)>0$. Since $\operatorname{Res}_{M}(W) \subseteq A$ and $\pi_{1 \mid A}$ is an embedding with linearly independent image, we obtain a contradiction.

(a2) Assume $E=\varnothing$, i.e., assume $W \subset H_{1} \cup H_{2}$. By step (a1) we may also assume $W \subseteq H_{1} \cup H_{3}$ with $H_{3} \in\left|\mathcal{I}_{u}\left(\varepsilon_{3}\right)\right|$. Set $\left\{M_{1}\right\}:=\left|\mathcal{I}_{\{o, u\}}\left(\varepsilon_{1}\right)\right|$ and take $M_{2} \in\left|\mathcal{I}_{0}\left(\varepsilon_{2}\right)\right|$ and $M_{3} \in\left|\mathcal{I}_{0}\left(\varepsilon_{3}\right)\right|$. Since $A \subset\left(M_{1} \cup M_{2}\right) \cap\left(M_{1} \cup M_{3}\right)$, we also obtain $Z \subset$ $M_{1} \cup M_{2}$ and $Z \subset M_{1} \cup M_{3}$. Assume that either $Z \cap H_{1}=\varnothing$ or $Z \cap M_{1}=\varnothing$, say $Z \cap H_{1}=\varnothing$. We get $Z \subset H_{2}$. The residual exact sequence of $H_{2}$ and ([33], Lemma 5.1) give a contradiction because $\operatorname{deg}\left(\pi_{1}(A)\right)=3$ and $\pi_{1}(A)$ is linearly independent. Assume that either $Z \subset H_{1}$ or $Z \subset M_{1}$, say $Z \subset H_{1}$. The residual exact sequence of $H_{1}$ gives a contradiction. Thus, $\operatorname{deg}\left(Z \cap H_{1}\right)=\operatorname{deg}\left(Z \cap M_{1}\right)=$ 1. Using $H_{1}$ we obtain $h^{1}\left(\mathcal{I}_{\operatorname{Res}_{H_{1}}(W)}\left(\hat{\varepsilon}_{1}\right)\right)>0$ with $\operatorname{Res}_{H_{1}}(W)$ the union of $u$ and a point of $A_{\text {red }}$, call it $e$. Since $h^{1}\left(\mathcal{I}_{\operatorname{Res}_{H_{1}}(W)}\left(\hat{\varepsilon}_{1}\right)\right)>0, \pi_{i}(u)=\pi_{i}(e)$ for all $i>1$. Take $R \in\left|\mathcal{I}_{u}\left(\varepsilon_{3}\right)\right|$. Using $R$ we obtain $h^{1}\left(\mathcal{I}_{v \cup \operatorname{Res}_{R}(Z)}\left(\hat{\varepsilon}_{3}\right)\right)>0$. Using $H_{2}$ we obtain $h^{1}\left(\mathcal{I}_{v \cup \operatorname{Res}_{H_{2}}(Z)}\left(\hat{\varepsilon}_{2}\right)\right)>0$. Thus, $\pi_{i}\left(\operatorname{Res}_{H_{2}}(Z)\right) \subseteq \pi_{i}(v)$ for all $i \neq 2$. Since $\operatorname{dim}\left|\mathcal{O}_{Y}\left(\varepsilon_{2}\right)\right|+\operatorname{dim}\left|\mathcal{O}_{Y}\left(\varepsilon_{3}\right)\right| \geq 2$, there are $T \in\left|\mathcal{O}_{Y}\left(\varepsilon_{2}\right)\right|$ and $T^{\prime} \in\left|\mathcal{O}_{Y}\left(\varepsilon_{2}\right)\right|$ such that $Z \subset T \cup T^{\prime}$. First assume $A \nsubseteq T \cup T^{\prime}$. Since $A \cap Z=\varnothing$, ([33], Lemma 5.1) gives $h^{1}\left(\mathcal{I}_{\operatorname{Res}_{T \cup T^{\prime}}(A)}(1,0,0, \ldots)\right)>0$. Since $\operatorname{deg}\left(\pi_{1}(A)\right)=3$ and $\pi_{1}(A)$ is linearly independent, we obtained a contradiction. Now assume $A \subset T \cup T^{\prime}$. If $Z \subset T$ (resp. $Z \subset T^{\prime}$ ) we may take instead of $T^{\prime}$ (resp. $T$ ) a general element of its complete linear system and obtain a contradiction, because $Y$ is the minimal multiprojective space containing $A$. Thus, $T \cap Z \neq \varnothing$ and $T^{\prime} \cap Z \neq \varnothing$. One of the two divisors $T$ or $T^{\prime}$, say $T$, contains $v$. Set $\{a\}:=\operatorname{Res}_{T}(Z)$. Please note that $a \in T^{\prime}$. We obtain $h^{1}\left(\mathcal{I}_{\{u, a\}}\left(\hat{\varepsilon}_{2}\right)\right)>0$, i.e., $\pi_{i}(a)=\pi_{i}(u)$ for all $i \neq 2$. If $a=e$ we obtain $a=u$, a contradiction. Please note that $a=e$ if $Z$ is connected. Assume $a \neq e$ and hence $Z=\{a, e\}$. Since $A \nsubseteq T, u \in T^{\prime}$. Hence $\pi_{3}(a)=\pi_{3}(u)$. Since $a \neq e$, we obtain $\operatorname{Res}_{T^{\prime}}(W) \subseteq v$. Hence the residual exact sequence of $T^{\prime}$ gives a contradiction.

(b) Assume $Y=\left(\mathbb{P}^{1}\right)^{k}$. All cases with $k \leq 3$ are listed. Thus, we assume $k \geq 4$. Let $e_{1}$ be the maximal integer such that $e_{1}=\operatorname{deg}(H \cap W)$ for some $i \in\{1, \ldots, k\}$ and some 
$H \in\left|\mathcal{O}_{Y}\left(\varepsilon_{i}\right)\right|$. With no loss of generality we may assume $i=1$. Set $W_{1}:=\operatorname{Res}_{H}(W)$. Let $e_{2}$ be the maximal integer such that $e_{2}=\operatorname{deg}\left(M \cap W_{1}\right)$ for some $i \in\{2, \ldots, k\}$ and some $M \in\left|\mathcal{O}_{Y}\left(\varepsilon_{i}\right)\right|$. With no loss of generality we may assume $i=2$. Set $W_{2}:=\operatorname{Res}_{H}\left(W_{1}\right)$. Obviously $e_{1} \geq e_{2}$. Since $Y$ is the minimal multiprojective space containing $A, 1 \leq i \leq 4$.

(b1) Assume $e_{1}=4$. Quoting ([33], Lemma 5.1) we obtain a contradiction.

(b2) Assume $e_{1}=2$. Thus, $1 \leq e_{2} \leq 2$. If $e_{2}=2$ applying ([33], Lemma 5.1) to $H \cup M$ we obtain a contradiction. Assume $e_{2}=1$. The definition of $e_{1}$ gives that each $\pi_{i \mid W_{1}}, i>2$, is an embedding. Fix $D \in\left|\mathcal{O}_{Y}\left(\varepsilon_{3}\right)\right|$ intersecting $W_{2}$. Please note that $\operatorname{deg}\left(\operatorname{Res}_{D}\left(W_{2}\right)\right)=1$. The residual exact sequence of $H \cup M \cup D$ and ([33], Lemma 5.1) gives a contradiction.

(b3) Assume $e_{1}=1$. The definition of $e_{1}$ gives that each $\pi_{i \mid W}$ is an embedding. Take $D \in\left|\mathcal{O}_{Y}\left(\varepsilon_{3}\right)\right|$ such that $D \cap W_{2} \neq \varnothing$ and set $W_{3}:=\operatorname{Res}_{D}\left(W_{2}\right)$. Please note that $\operatorname{deg}(D \cap W)=1$ and that $\operatorname{deg}\left(W_{3}\right)=2$. Since $\pi_{4 \mid W_{2}}$ is an embedding, $h^{1}\left(\mathcal{I}_{W_{2}}(0,0,0,1, \ldots)\right)=0$, contradicting ([33], Lemma 5.1).

(b4) Assume $e_{1}=3$. If $e_{2}=1$ quoting ([33], Lemma 5.1) we obtain a contradiction. Now assume $e_{2}=2$, i.e., assume $W \subset H \cup M$. If there are $i \in\{3, \ldots, k\}$ and $D \in\left|\mathcal{O}_{Y}\left(\varepsilon_{i}\right)\right|$ with $\operatorname{deg}\left(W_{1} \cap D\right)=1$, then quoting ([33], Lemma 5.1) with respect to $H \cup D$ we obtain a contradiction. Thus, we may assume $\operatorname{deg}\left(\pi_{i}\left(W_{1}\right)\right)=1$ for all $i \geq 2$. Hence $\pi_{1 \mid W_{1}}$ is an embedding. Set $E:=\operatorname{Res}_{M}(W)$. Since in step (b1) we excluded the case $e_{1}=4$ and $W_{1} \subset M$, we have $2 \leq \operatorname{deg}(W \cap M) \leq 3$ and hence $2 \leq \operatorname{deg}(E) \leq 3$. By assumption $E \subset H$ and hence $\operatorname{deg}\left(\pi_{1}(E)\right)=1$.

(b4.1) Assume $\operatorname{deg}(E)=2$. Using $M$ instead of $H$ in the first part of step (b4) we obtain $\operatorname{deg}\left(\pi_{i}(E)\right)=1$ for all $i \neq 2$. Take $H_{i} \in\left|\mathcal{O}_{Y}\left(\varepsilon_{i}\right)\right|, 3 \leq i \leq k$, containing $E$. We obtain $W \subset M+H_{i}$. We also have $W \subset H \cup M$. Please note that the set $M \cap H \cap H_{3} \cap \cdots \cap H_{k}$ is a point and that $v\left(M \cap H_{3} \cap\right.$ $\left.\cdots \cap H_{k}\right)$ is a line of the Segre variety $v(M)$. We obtain $W_{\text {red }} \subset M$. Since $\operatorname{deg}(W \cap M)=3$, we obtain\# $W_{\text {red }}=3$, i.e., $Z$ is not reduce. Using $H$ instead of $M$ we obtain $W_{\text {red }} \subset H \cap M$. Using $M$ and $H_{i}, 3 \leq i \leq k$, we obtain $W_{\text {red }}$ is contained in the point $H \cap M \cap H_{3} \cap \cdots \cap H_{k}$, absurd.

(b4.2) Assume $\operatorname{deg}(E)=3$. In the set-up of step (b4.1) we may also assume that $E \cap H_{i}=\varnothing$ for all $i=3, \ldots, k$. Set $H_{2}:=M$. Let $w \subset E$ be a degree 2 scheme. Since $v$ is an embedding, there is $i_{w} \in\{2, \ldots, k\}$ such that $\pi_{i_{w} \mid w}$ is an embedding. Let $M_{w}$ be an element of $\left|\mathcal{O}_{Y}\left(\varepsilon_{i_{w}}\right)\right|$ containing a point of $w_{\text {red }}$. Fix $i \in\{2, \ldots, k\} \backslash\left\{i_{w}\right\}$ and set $F:=\operatorname{Res}_{H_{i}+M_{w}}(W)$. Since $\operatorname{deg}(F \cap w)=1,1 \leq \operatorname{deg}(F) \leq 2$. By ([33], Lemma 5.1) we first obtain $\operatorname{deg}(F)=2$ and then $\operatorname{deg}\left(\pi_{h}(F)\right)=1$ for all $h \notin\left\{i, i_{w}\right\}$. Either $E$ is the union of 3 points, say $E=\{a, b, c\}$, or the union of a point $a$ and an arrow $z$.

(b4.2.1) Assume $E=\{a, b, c\}$. Thus, $E \neq A$. Take $w:=\{b, c\}$. Taking $M_{w}$ containing $c$ we obtain $\pi_{h}(a)=\pi_{h}(b)$ for all $h \notin\left\{i, i_{w}\right\}$. Taking $M_{w}$ containing $b$ we obtain $\pi_{h}(a)=\pi_{h}(c)$ for all $h \notin$ $\left\{i, i_{w}\right\}$. Thus, $\operatorname{deg}\left(\pi_{h}(E)\right)=1$ for all $h \notin\left\{i, i_{w}\right\}$. Varying $i$ we obtain $\operatorname{deg}\left(\pi_{h}(E)\right)=1$ for all $h \neq i_{w}$. In this case $\langle v(E)\rangle$ is a line contained in the $i_{w}$-ruling of the Segre $v(Y)$. Thus, $v(E)$ is linearly dependent. Since $k>2$, Proposition 1 gives $q \notin v(Y)$. Since $q \in\langle v(A)\rangle \cap\langle v(Z)\rangle$ and $q \notin\left\langle v\left(A^{\prime}\right)\right\rangle$ for any $A^{\prime} \subsetneq A$, we obtain $h^{1}\left(\mathcal{I}_{W}(1, \ldots, 1)\right) \geq 2$. Since $v(A)$ is linearly independent, $h^{1}\left(\mathcal{I}_{W}(1, \ldots, 1)\right)=2$. Thus, $v(Z)$ is contained in the plane, $\langle v(A)\rangle$, which also contains the line $\langle v(E)\rangle \subset v(Y)$. Since $q \notin\left\langle v\left(Z^{\prime}\right)\right\rangle$ for any $Z^{\prime} \subsetneq Z, q \notin\left\langle v\left(A^{\prime}\right)\right\rangle$ for any $A^{\prime} \subsetneq A$ and $Z \cap A=\varnothing$, $h^{1}\left(\mathcal{I}_{W^{\prime}}(1, \ldots, 1)\right) \leq 1$ for all $W^{\prime} \neq W$. Thus, $\langle v(E)\rangle \cap v(W)=E$ (scheme-theoretically). Since $v(Y)$ contains no plane $\langle v(A)\rangle \nsubseteq$ $v(Y)$. Since $v(W) \subset\langle v(A)\rangle$ and $v(Y)$ is scheme-theoretically cut 
out by quadrics, $v(Y) \cap\langle v(A)\rangle$ is the union of 2 lines. Since $Y$ is the minimal multiprojective space containing $A$, we obtain $k=2$ (([29], Proposition 5.2) or ([30], Proposition 1.1) or Theorem 1), a contradiction.

(b4.2.2) Assume $E=z \cup\{a\}$ and set $\{b\}:=z_{\text {red }}$. Take $w=z$. We obtain $\pi_{h}(a)=\pi_{h}(b)$ for all $h \notin\left\{i, i_{w}\right\}$. Varying $i$ we obtain $\pi_{h}(a)=$ $\pi_{h}(b)$ for all $h \neq i_{w}$. Take $w=\{a, b\}$, but call $i_{w^{\prime}}$ the integer associated with this degree 2 scheme. We obtain $\pi_{h}(a)=\pi_{h}(b)$ for all $h \neq i_{w^{\prime}}$. Thus, $i_{w}=i_{w^{\prime}}$. Thus, $\langle\{a, b\}\rangle$ is a line contained in the $w$-th ruling of $v(Y)$. If $v(E) \subset\langle\{a, b\}\rangle$ we conclude as in step (b4.2.1). Assume $v(E) \nsubseteq\langle\{a, b\}\rangle$. Either $z=v$ or $Z$ is connected and $Z=z$. Take $h \neq i_{w}$ and $D \in\left|\mathcal{I}_{a}\left(\varepsilon_{h}\right)\right|$. By construction $\operatorname{deg}\left(W \cap\left(H_{i_{w}} \cup D\right)\right)=4$. Thus, $h^{1}\left(\mathcal{I}_{\operatorname{Res}_{H_{i w}} \cup D}\right)=0$, contradicting ([33], Lemma 5.1).

Remark 11. Take $Y:=\left(\mathbb{P}^{1}\right)^{3}$ and hence $r=7$. A general $q \in \mathbb{P}^{7}$ is contained in $\langle v(A)\rangle$ for $\infty^{4}$ sets $A \subset Y$ such that $\# A=3, \infty^{3}$ unions $A$ of an arrow and a point and for exactly one set $A \subset Y$ such that $\# A=2$. A general $q$ in the tangential variety of $v(Y) \subset \mathbb{P}^{7}$ is contained in $\langle v(A)\rangle$ for $\infty^{4}$ sets $A \subset Y$ such that $\# S=3, \infty^{3}$ unions $A$ of an arrow and a point and exactly one arrow, but no set $A$ with cardinality 2.

Example 3. Take $Y=\mathbb{P}^{2} \times \mathbb{P}^{1} \times \mathbb{P}^{1}$ and take $q \in \mathbb{P}^{r}$ such that $r_{v(Y)}(q)=3$ and there is $H \in\left|\mathcal{O}_{Y}(0,1,1)\right|$ with $q \in\langle v(H)\rangle$. This case covers cases (4) and (5) of ([26], Theorem 7.1), case (4) being the case H irreducible, while case (5) being the case H reducible. If $H$ is irreducible (resp. it is reducible), then $q$ is in the linear span of $\infty^{3}$ (resp. $\left.\infty^{4}\right)$ subsets of $v(Y)$ with cardinality 3. For many $q \in \mathbb{P}^{r}$ we have $q \in\langle v(A)\rangle$ for some union $A$ of an arrow and a point.

Lemma 3. Let $E \subset Y$ be a planar double point. Set $\{o\}:=E$ and write $E=v \cup w$ with $v$ and $w$ arrows with $o$ as their reduction. Fix $u, z \in Y \backslash\{o\}$ such that $u \neq z$ and set $W:=E \cup\{u, z\}$ and $A:=v \cup\{u\}$. Assume that $Y$ is the minimal multiprojective space containing $v \cup\{u\}$, that $\langle v(A)\rangle \cap\langle v(w \cup\{z\})\rangle$ contains a point $q$ and that $q \notin\left\langle v\left(A^{\prime}\right)\right\rangle$ for any $A^{\prime} \subsetneq A$. Then $k \leq 2$ and $n_{1}=n_{2}=1$ if $k=2$.

Proof. By assumption $q \in\langle v(E)\rangle \cap\langle\{v(u), v(z)\}\rangle$. Thus, there is an arrow $\tau \subset E$ such that $q \in\langle v(\tau)\rangle \cap\langle v(w \cup\{z\})\rangle$. Proposition 1 shows that the minimal multiprojective space $Y^{\prime}$ containing $W^{\prime}:=\tau \cup\{u, z\}$ is either a projective space or $\mathbb{P}^{1} \times \mathbb{P}^{1}$. If $v \subset Y^{\prime}$, then the lemma is true. Assume $v \nsubseteq Y^{\prime}$. Since $\left\langle v\left(Y^{\prime}\right)\right\rangle \cap v(Y)=v\left(Y^{\prime}\right)$, we obtain $\langle v(A)\rangle=\langle v(\{o, u\}\rangle$, a contradiction.

Example 4. Assume $k \geq 3$. We do the construction for the first 2 positive integers, but the case in which we take any two distinct elements of $\{1, \ldots, k\}$ is similar. Fix arrows $u^{\prime}, v^{\prime} \subset Y^{\prime}:=\mathbb{P}^{1} \times \mathbb{P}^{1}$ such that $u^{\prime} \cap v^{\prime}=\varnothing$ and $Y^{\prime}$ is the minimal multiprojective space containing $u^{\prime} \cup v^{\prime}$, but that $\left\langle v\left(u^{\prime} \cup v^{\prime}\right)\right\rangle$ is a plane. Thus, $\left\langle v\left(u^{\prime}\right)\right\rangle \cap\left\langle v\left(v^{\prime}\right)\right\rangle$ is a single point. Fix $n_{1} \in\{1,2\}$ and $n_{2} \in\{1,2\}$. Set $Y:=\mathbb{P}^{n_{1}} \times \mathbb{P}^{n_{2}} \times\left(\mathbb{P}^{1}\right)^{k-2}$ with $Y^{\prime}$ embedded in the first two factors of $Y$. Fix $a_{i}, o_{i} \in \mathbb{P}^{1}$, $3 \leq i \leq k$, such that $a_{i} \neq o_{i}$ for all $i$. Set $u:=v^{\prime} \times\left\{\left(a_{3}, \ldots, a_{k}\right)\right\}$. Thus, $\langle v(u)\rangle \cap\langle v(v)\rangle$ is $a$ single point, $q^{\prime}$. Fix $o_{1} \in \mathbb{P}^{n_{1}}$ and $o_{2} \in \mathbb{P}^{n_{2}}$ with the restriction that $o_{1} \notin \mathbb{P}^{1}$ if $n_{1}=2$ and $o_{2} \notin \mathbb{P}^{1}$ if $n_{2}=2$. Set $\left.o:=\left(o_{1}, \ldots, o_{k}\right)\right\}$. Please note that $\langle v(u \cup\{o\})\rangle$ is the line spanned by $q^{\prime}$ and $v(o)$. Please note that $Y$ is the minimal multiprojective space containing $u \cup\{o\}$ if and only if one of the following conditions holds:

1. $Y^{\prime}$ is the minimal multiprojective space containing $u^{\prime}$.

2. $Y^{\prime}$ is not the minimal multiprojective space containing $u^{\prime}$, i.e., $\operatorname{deg}\left(\pi_{i}\left(u^{\prime}\right)=1\right.$ for exactly one $i \in\{1,2\}$; in this case we require $n_{i}=1$ and $\pi_{i}\left(u^{\prime}\right) \neq o_{i}$. 
Example 5. The construction done in Example 4 works if instead of the arrow $v^{\prime}$ we take 2 distinct points of $Y^{\prime}$.

Example 6. Assume $k \geq 3$. We do the construction for the first 2 positive integers, but the case in which we take any two distinct elements of $\{1, \ldots, k\}$ is similar. Set $Y^{\prime}:=\mathbb{P}^{1} \times \mathbb{P}^{1}$ and fix $a \in Y^{\prime}$. Let $L$ and $L^{\prime}$ be the elements of $\left|\mathcal{O}_{Y^{\prime}}(1,0)\right|$ and $\left|\mathcal{O}_{Y^{\prime}}(0,1)\right|$ containing $a$. Let $u^{\prime}$ (resp. $\left.v^{\prime}\right)$ be the arrow of $L$ (resp. $L^{\prime}$ ) containing $a$. Fix $n_{1} \in\{1,2\}$ and $n_{2} \in\{1,2\}$. Set $Y:=\mathbb{P}^{n_{1}} \times \mathbb{P}^{n_{2}} \times\left(\mathbb{P}^{1}\right)^{k-2}$ with $Y^{\prime}$ embedded in the first two factors of $Y$. Fix $a_{i}, o_{i} \in \mathbb{P}^{1}$, $3 \leq i \leq k$, such that $a_{i} \neq o_{i}$ for all $i$. Set $u:=u^{\prime} \times\left\{\left(a_{3}, \ldots, a_{k}\right)\right\}$ and $v:=u^{\prime} \times\left\{\left(a_{3}, \ldots, a_{k}\right)\right\}$. Fix $o_{1} \in \mathbb{P}^{n_{1}}$ and $o_{2} \in \mathbb{P}^{n_{2}}$ with the restriction that $o_{1} \notin \mathbb{P}^{1}$ if $n_{1}=2$ and $o_{2} \notin \mathbb{P}^{1}$ if $n_{2}=2$. Set $\left.o:=\left(o_{1}, \ldots, o_{k}\right)\right\}$. Please note that $\langle v(u \cup\{o\})\rangle \cap\langle v(v \cup\{o\})\rangle$ is the line spanned by $v(a)$ and $v(o)$ and it is not contained in $v(Y)$. We obtain that a general $q \in\langle\langle v(u \cup\{o\})\rangle \cap\langle v(v \cup\{o\})\rangle$ has rank 2. Please note that $Y$ is the minimal multiprojective space containing $u \cup\{o\}$ if and only if $n_{2}=1$ and $\pi_{2}(u) \neq o_{2}$.

Example 7. Take either $Y=\left(\mathbb{P}^{1}\right)^{k}$ or $Y=\mathbb{P}^{2} \times\left(\mathbb{P}^{1}\right)^{k-1}, k>1$. Fix $o \in Y$ and take an arrow $v \subset Y$ such that $v_{\text {red }}=\{o\}$ and $\pi_{i}(v)=\pi_{i}(o)$ for all $i>1$. Please note that $\langle v(v)\rangle=v(L)$ with $L=\mathbb{P}^{1} \times\left(o_{2}, \ldots, o_{n}\right) \subset Y$. Fix $u \in Y$ such that $\pi_{i}(u) \neq \pi_{i}(o)$ for $i=2, \ldots, k$. If $Y=\mathbb{P}^{2} \times\left(\mathbb{P}^{1}\right)^{k-1}$ assume $\pi_{1}(o) \notin \pi_{1}(L)$. Set $A:=v \cup u$. Please note that $Y$ is the minimal multiprojective space containing $A$. Each $q \in\langle v(A)\rangle$ has tensor rank at most 2 , because there is $a_{q} \in L$ such that $q \in\left\langle v\left(\left\{a_{q}, u\right\}\right)\right\rangle$.

Example 8. Take either $Y=\left(\mathbb{P}^{1}\right)^{k}$ or $Y=\mathbb{P}^{2} \times\left(\mathbb{P}^{1}\right)^{k-1}, k>1$. Fix $o, u \in Y$ such that $o \neq u$ and $\pi_{i}(o)=\pi_{i}(u)$ for all $i>1$. Take an arrow $v \subset Y$ such that $v_{\text {red }}=\{o\}$ and $\operatorname{deg}\left(\pi_{i}(v)\right)=2$ for all $i>1$. Please note that $\langle v(\{o, u\})\rangle=v(L)$ with $L=\mathbb{P}^{1} \times\left(o_{2}, \ldots, o_{n}\right) \subset Y$. If $Y=$ $\mathbb{P}^{2} \times\left(\mathbb{P}^{1}\right)^{k-1}$ assume $\pi_{1}(v) \notin \pi_{1}(L)$. Set $A:=v \cup u$. Please note that $Y$ is the minimal multiprojective space containing $A$ and that $\langle v(A)\rangle=\left\langle v\left(v \cup o^{\prime}\right)\right\rangle$ for all $o^{\prime} \in L$.

Lemma 4. Take $Y=\mathbb{P}^{2} \times \mathbb{P}^{2} \times \mathbb{P}^{n_{3}} \times \cdots \times \mathbb{P}^{n_{k}}, k \geq 3$. Theorem 3 is true for $Y$ and all $B$ such that $\operatorname{deg}(B)=3$ and $A \cap B=\varnothing$.

Proof. Assume the existence of $B \subset Y$ such that $B$ is either the union of an arrow and a point or the union of 3 distinct points, $B \neq A$ and $q \in\langle v(B)\rangle$. Set $W:=A \cup B$. By Propositions 1 and 2 we may assume that $v(B)$ irredundantly spans $q$. Since $\operatorname{dim}\left|\mathcal{O}_{Y}\left(\varepsilon_{2}\right)\right|+$ $\operatorname{dim}\left|\mathcal{O}_{Y}\left(\varepsilon_{3}\right)\right| \geq 3$, there is $H^{\prime} \in\left|\mathcal{O}_{Y}\left(\varepsilon_{2}\right)\right|$ and $H^{\prime \prime} \in\left|\mathcal{O}_{Y}\left(\varepsilon_{3}\right)\right|$ such that $H:=H^{\prime} \cup H^{\prime \prime}$ contains $B$. Since $h^{0}\left(\mathcal{O}_{\mathcal{Y}}\left(\uparrow_{\infty}\right)\right)=\operatorname{deg}(\mathcal{A})$ and $Y$ is the minimal multiprojective space containing $A, h^{1}\left(\mathcal{I}_{A}\left(\varepsilon_{1}\right)\right)=0$. Thus, the residual exact sequence of $H$ and ([33], Lemma 5.1) give $W \subset H$. This is true for all $H^{\prime}, H^{\prime \prime}$ whose union contains $B$. Since $Y$ is the minimal multiprojective space containing $A, \pi_{2}(A)$ spans $\mathbb{P}^{2}$. Thus, $\operatorname{deg}\left(H^{\prime} \cap A\right) \leq 2$. We obtain that $\pi_{2}(B)$ spans $\mathbb{P}^{2}$. Thus, we obtain that the lines of $\mathbb{P}^{2}$ spanned by degree 2 subschemes of $A$ and $B$ are the same. We also obtain that $\pi_{3 \mid B}$ is an embedding and then we get that $\pi_{3 \mid A}$ is an embedding. Since $A \cap B=\varnothing$, ([33], Lemma 5.1) and the residual exact sequence of $H^{\prime \prime}$ gives $h^{1}\left(\mathcal{I}_{\operatorname{Res}_{H^{\prime}}(W)}\left(\hat{\varepsilon}_{2}\right)\right)>0$. Please note that $\operatorname{Res}_{H^{\prime}}(W)$ is a degree 2 reduced scheme, one of its 2 points being in $A_{\text {red, }}$, while the other one is an element of $B_{\text {red }}$. Since $\pi_{3 \mid B}$ and $\pi_{3 \mid A}$ are embeddings with the same set-theoretic image, $B$ is the union of an arrow and a point, say $B=v^{\prime} \cup\left\{u^{\prime}\right\}$ with $\pi_{3}\left(u^{\prime}\right)=\pi_{3}(u)$. Taking $H^{\prime \prime}$ containing $\pi_{2}\left(u^{\prime}\right)$ we obtain $\pi_{j}\left(u^{\prime}\right)=\pi_{j}(u)$ for all $j \neq 2$. Using $\left|\mathcal{O}_{Y}\left(\varepsilon_{1}\right)\right|$ and $\left|\mathcal{O}_{Y}\left(\varepsilon_{3}\right)\right|$ in the same way we obtain $\pi_{j}\left(u^{\prime}\right)=\pi_{j}(u)$ for all $j \neq 1$, contradicting the assumption $A \cap B=\varnothing$.

Lemma 5. Take $Y=\mathbb{P}^{2} \times\left(\mathbb{P}^{1}\right)^{k-1}, k \geq 4$. Theorem 3 is true for $Y$ and all $B$ such that $\operatorname{deg}(B)=3$ and $A \cap B=\varnothing$.

Proof. Assume the existence of $B \subset Y$ such that $B$ is either the union of an arrow and a point or the union of 3 distinct points, $B \neq A$ and $q \in\langle v(A)\rangle$. Set $W:=A \cup B$. 
By Propositions 1 and 2 we may assume that $v(B)$ irredundantly spans $q$. Fix $i \in\{2, \ldots, k\}$. Mimicking the proof of Lemma 4 using $\left|\mathcal{O}_{Y}\left(\varepsilon_{1}\right)\right|$ and $\left|\mathcal{O}_{Y}\left(\varepsilon_{i}\right)\right|$ instead of $\left|\mathcal{O}_{Y}\left(\varepsilon_{2}\right)\right|$ and $\left|\mathcal{O}_{Y}\left(\varepsilon_{3}\right)\right|$ and doing it for all $i$ we obtain that $B$ is the union of a point $u^{\prime}$ and an arrow $v^{\prime}$ that each $\pi_{h \mid B}$ and $\pi_{h \mid A}$ are embeddings with the same images, that $\pi_{1}(B)$ spans $\mathbb{P}^{2}$ and that $\pi_{j}(u)=\pi_{j}\left(u^{\prime}\right)$ and $\pi_{j}(v)=\pi_{j}\left(v^{\prime}\right)$ for all $j>1$. Set $o^{\prime}:=v_{\text {red }}^{\prime}$. We proved that $\pi_{h}\left(o^{\prime}\right)=\pi_{h}(o)$ for all $h>1$. Take $M \in \mid \mathcal{O}_{Y}\left(\varepsilon_{2}\right)$ containing $o^{\prime}$ and $M^{\prime} \in\left|\mathcal{O}_{Y}\left(\varepsilon_{3}\right)\right|$. Please note that $\operatorname{Res}_{M \cup M^{\prime}}(W)=\left\{o, o^{\prime}\right\}$. Since $A \cap B=\varnothing$, ([33], Lemma 5.1) and the residual exact sequence of $M \cup M^{\prime}$ give $\pi_{1}\left(o^{\prime}\right)=\pi_{1}(o)$. Thus, $A \cap B \neq \varnothing$, a contradiction.

Remark 12. Assume that $Y$ is the minimal multiprojective space containing $A=v \cup\{u\}$, $o:=v_{\text {red }}$, and that $k \geq 2$. Fix $i \in\{1, \ldots, k\}$ and assume that $\eta_{i \mid A}$ is not an embedding. Since $Y$ is the minimal multiprojective space containing $A$, we obtain $\operatorname{deg}\left(\eta_{1}(A)\right)=2, n_{h}=1$ for all $h \neq i$ and that $\pi_{i \mid A}$ is an embedding. Since $\pi_{i \mid A}$ is an embedding, we obtain that $\eta_{h \mid A}$ is an embedding for all $h \neq i$.

(a) Assume $\eta_{i}(A)=\eta_{i}\left(\left\{o, o^{\prime}\right\}\right)$. In this case $\eta_{i}(v)=\eta_{i}(o)$. We obtain that $\langle v(v)\rangle$ is a line contained in $Y$. Thus, all points of $\langle v(A)\rangle$ have tensor rank at most 2 . This is Example 7.

(b) Assume $\eta_{i}(A)=\eta_{i}(v)$. In this case $\pi_{h}(u)=\pi_{h}(o)$ for all $h \neq i$. Thus, $\langle v(\{0, u\})\rangle$ is a line contained in the $i$-th ruling of the Segre $v(Y)$, say $\langle v(\{0, u\})\rangle=v(R)$. For each $u^{\prime} \in R \backslash\{0\}$ we have $\langle v(A)\rangle=\left\langle v\left(v \cup u^{\prime}\right)\right\rangle$. In particular uniqueness fails for each $q$ irredundantly spanned by $v(A)$. This case obviously occurs both with $n_{i}=1$ and with $n_{i}=2$. This is Example 8 .

(c) Fix a set $S \subset Y$ such that $\# S=3$ and $Y$ is the minimal multiprojective space containing $Y$. Assume the existence of $i \in\{1, \ldots, k\}$ such that $\eta_{i \mid S}$ is not injective. By ([26], Remark 1.10) each point of $\langle v(S)\rangle$ has tensor rank at most 2.

Since $v(A)$ is linearly independent, there is $B^{\prime} \subseteq B$ such that $v\left(A \cup B^{\prime}\right)$ is linearly dependent and $B^{\prime}$ is minimal with this property. If $B^{\prime} \neq B$ we obtain $\langle v(A)\rangle \cap$ From now on

Lemma 6. Take $Y=\left(\mathbb{P}^{1}\right)^{k}, k \geq 5$. Theorem 3 is true for $Y$ and all $B$ such that $\operatorname{deg}(B)=3$ and $A \cap B=\varnothing$.

Proof. Assume the existence of $B \subset Y$ such that $B$ is either the union of an arrow and a point or the union of 3 distinct points, $B \neq A$ and $q \in\langle v(A)\rangle$. Set $W:=A \cup B$. By Propositions 1 and 2 we may assume that $v(B)$ irredundantly spans $q$. If $B$ contains an arrow, then $\# W_{\text {red }}=4$. If $B$ is formed by 3 distinct points, then $\# W_{\text {red }}=5$.

(a) Assume for the moment that $Y$ is not the minimal multiprojective space containing $B$. Thus, there is $i \in\{1, \ldots, k\}$ and $H \in\left|\mathcal{O}_{Y}\left(\varepsilon_{i}\right)\right|$ such that $B \subset H$ and hence $\operatorname{Res}_{H}(W) \subseteq$ $A$. Since $A \cap B=\varnothing$, ([33], Lemma 5.1) gives $h^{1}\left(\mathcal{I}_{\operatorname{Res}_{H}(W)}\left(\hat{\varepsilon}_{1}\right)\right)=0$. Since $A \subseteq$ $\operatorname{Res}_{H}(W)$, either $\eta_{i \mid A}$ is not an embedding or $A=\operatorname{Res}_{H}(W)$ and $\operatorname{deg}\left(\pi_{j}(A)\right)=1$ for $k-2$ indices $j$, contradicting the minimality of $Y$. Thus, $Y$ is the minimal multiprojective space containing $B$. If there is $i \in\{1, \ldots, k\}$ such that $\eta_{i \mid B}$ is not an embedding, we apply Remark 12 to $B$.

(b) By assumption we are not as in Example 7 or 8 for $A$ or $B$. Thus, we may assume that all $\eta_{i \mid B}$ and all $\eta_{i \mid A}$ are embeddings. There are 4 degree 2 schemes $E \subset W$ formed by one point of $A_{\text {red }}$ and one point of $B_{\text {red }}$. Since $k>4$, there is $i \in\{1, \ldots, k\}$ such that $\eta_{i \mid W}$ is an embedding. With no loss of generality we may assume $i=1$.

(b1) Assume that $\pi_{1 \mid W}$ is an embedding. Take $M \in\left|\mathcal{O}_{Y}\left(\varepsilon_{1}\right)\right|$ containing a reduced connected component $a$ of $B$. Please note that $\operatorname{Res}_{M}(W)=W \backslash\{a\}$. Since $A \subset W^{\prime}$ and $Y$ is the minimal multiprojective space containing $Y, Y_{1}$ is the minimal multiprojective space containing $\eta_{1}(A) \subset \eta_{1}\left(\operatorname{Res}_{M}(W)\right)$ and $v_{1}\left(\eta_{1}(A)\right)$ is linearly independent by Remark 7 . Since $\eta_{1 \mid W}$ is an embedding, $\eta_{1 \mid \operatorname{Res}_{H}(W)}$ is an embedding. Thus, $h^{1}\left(\mathcal{I}_{\left.\operatorname{Res}_{H}(W)\right)}\left(\hat{\varepsilon}_{1}\right)\right)=h^{1}\left(Y_{1}, \mathcal{I}_{\eta_{1}\left(\operatorname{Res}_{H}(W)\right.}(1, \ldots, 1)\right)$. Since $A \cap B=\varnothing$ and $\eta_{1 \mid W}$ is an embedding, ([33], Lemma 5.1) gives 
$h^{1}\left(Y_{1}, \mathcal{I}_{\eta_{1}(W \backslash\{a\})}(1, \ldots, 1)\right)>0$. Since $k \geq 3$ and $Y$ is the minimal multiprojective space containing $Y, v_{1}(A)$ is linearly independent by ([26], Lemma 4.4). Let $W_{1} \subseteq \eta_{1}(W \backslash\{a\})$ the minimal subscheme of $\eta_{1}(W \backslash\{a\})$ containing $A$ such that $h^{1}\left(Y_{1}, \mathcal{I}_{W_{1}}(1, \ldots, 1)\right)>0$. If $\operatorname{deg}\left(W_{1}\right)=4\left(\right.$ resp. $\left.\operatorname{deg}\left(W_{1}\right)=5\right)$, Proposition 1 (resp. Proposition 2) gives that $\eta_{1}(A)$ depends on at most 2 (resp. 3) factors of $Y_{1}$. Thus, $A$ depends on at most 4 factors of $Y$, a contradiction.

(b2) Assume that $\pi_{1 \mid W}$ is not an embedding. If there is $M \in\left|\mathcal{O}_{Y}\left(\varepsilon_{1}\right)\right|$ such that $M \cap B \neq \varnothing$ and $M \cap A=\varnothing$, then we may repeat the proof of step (b1). Since $Y$ is the minimal multiprojective space containing $B$ by step (a), we conclude if there is $M \in\left|\mathcal{O}_{Y}\left(\varepsilon_{1}\right)\right|$ such that $M \cap A \neq \varnothing$ and $M \cap B=\varnothing$. Thus, we may assume $\pi_{1}(A)_{\text {red }}=\pi_{1} \mid(B)_{\text {red }}$. Since $A \cap B=\varnothing$ and $A \nsubseteq H$, ([33], Lemma 5.1) gives $h^{1}\left(\mathcal{I}_{\left.\operatorname{Res}_{H}(W)\right)}\left(\hat{\varepsilon}_{1}\right)\right)>0$. Since $\eta_{1 \mid W}$ is an embedding, $\eta_{\mid \operatorname{Res}_{H}(W)}$ is an embedding. Thus, $h^{1}\left(\mathcal{I}_{\left.\operatorname{Res}_{H}(W)\right)}\left(\hat{\varepsilon}_{1}\right)\right)=h^{1}\left(Y_{1}, \mathcal{I}_{\eta_{1}\left(\operatorname{Res}_{H}(W)\right.}(1, \ldots, 1)\right)$. Let $W_{1} \subseteq$ $\eta_{1}\left(\operatorname{Res}_{H}(W)\right)$ be a minimal subscheme of such that $h^{1}\left(Y_{1}, \mathcal{I}_{W_{1}}(1, \ldots, 1)\right)>0$. If $W_{1}=\eta_{1}\left(\operatorname{Res}_{H}(W)\right)$, then Proposition 2 gives that $\operatorname{Res}_{H}\left(W_{1}\right)$ depends on at most 3 factors of $Y$, one of them being the first one.

Example 9. Assume $Y=\left(\mathbb{P}^{1}\right)^{4}$, that $Y$ is the minimal multiprojective space containing A. Fix $q \in\langle v(A)\rangle$ with tensor rank $>2$. If $q$ has tensor rank 3 , then its tensor rank is evinced by $\infty^{1}$ sets $v(S)$ with $\# S=3$, because $\sigma_{3}(Y)$ is defective ([34-36]).

Observation 1: Assume that $\pi_{i \mid A}$ is an embedding for all $i=1,2,3,4$. There are $\infty^{1}$ morphisms $f_{i}: \mathbb{P}^{1} \rightarrow \mathbb{P}^{1}$ such that $f_{i}(0)=\pi_{i}(o)$ and $f_{i}(\infty)=\pi_{i}(u)$. Thus, we obtain $\infty^{4}$ different morphism $f: \mathbb{P}^{1} \rightarrow Y$ such that $f(0)=o$ and $f(\infty)=u$ and the linear spans of the images of $\left(20, \mathbb{P}^{1}\right)$ by these morphisms covert $=$ the projective tangent space $\mathbb{P} T_{0} Y$ except its 4 hyperplanes tangents to the hypersurfaces $\pi_{i}^{-1}(o)$. Hence $A \subset f\left(\mathbb{P}^{1}\right)$ for some $f$. Thus, $v(A)$ is contained in the rational normal curve $C:=v\left(f\left(\mathbb{P}^{1}\right)\right)$ of the 4 -dimensional space $\left\langle v\left(f\left(\mathbb{P}^{1}\right)\right)\right\rangle$. The point $q$ has cactus rank $\leq 3$ with respect to $C$. By Sylvester's theorem $r_{C}(q)=3$ and the $C$-rank is achieved by $\infty^{1}$ subsets of $C$ with cardinality 3.

Now assume that $\pi_{i \mid A}$ is not an embedding for some $i$, say for $i=1$. We extend $\Sigma_{3}^{0}(v(Y))$ taking instead of $S(v(Y), 3)$ the open subset $U$ of the irreducible component $\mathbb{H}(X, 3)$ of the Hilbert scheme of $v(Y)$ containing $S(v(Y), 3)$ and formed by degree 3 linearly independent schemes. Please note that $A \in U$ and that $A$ is limit of elements $A^{\prime} \in U$ such that each $A^{\prime}$ is a union of an arrow and a point and $\pi_{i}\left(A^{\prime}\right)$ is an embedding for all $i=1,2,3,4$ and all $A^{\prime} \neq A$. Use Observation 1 and ([32], Ex. II.3.22) to see that in this case $q$ is spanned by infinitely many $v\left(A^{\prime}\right)$. Since $A$ has 2 connected components, each nearby $A^{\prime}$ has at least 2 connected components, i.e., it is the union of either 3 points or a point and an arrow.

Proof of Theorem 3. If $\operatorname{deg}(B) \leq 2$, then we are in the set-up of Propositions 1 and 2. Thus, we may assume $\operatorname{deg}(B)=3$.

If $k=2$ we use Remark 10. Thus, we may assume $k \geq 3$.

If $A \cap B \neq \varnothing$ we use Proposition 2 and the examples listed in case (7) of the theorem. Thus, we may assume $A \cap B=\varnothing$.

If $n_{1}=n_{2}=2$ we use Lemma 4 .

If $n_{1}=2$ and $n_{1}=1$ we use Lemma 5 .

Example 9 consider the case $Y=\left(\mathbb{P}^{1}\right)^{4}$.

Lemma 6 exclude the case $Y=\left(\mathbb{P}^{1}\right)^{k}, k \geq 5$.

\section{Speculations on the Higher Derivatives and Uniqueness}

Fix integral and non-degenerate varieties $X \subset \mathbb{P}^{r}$ and $W \subset \mathbb{P}^{r}$. Set $n:=\operatorname{dim} X$ and $m:=\operatorname{dim} W$. Fix a finite set $S \subset X_{\text {reg }}, S \neq \varnothing, u \in X_{\text {reg }}$ and $v \in W_{\text {reg. }}$. Set $k:=\# S$. Suppose that $\operatorname{dim}\left\langle\cup_{o \in S}(2 o, X)\right\rangle<k(n+1)-1($ resp. $\operatorname{dim}\langle(2 u, X) \cup(2 v, W) \leq n+m)$, i.e., assume that $S$ is in the $k$-Terracini locus of $S$ (resp. the pair $(u, v)$ is in the Terracini locus of the 
join of $X$ and $W$ ). Thus, the differential of a certain map, call it $f$, is not injective at $S$ (resp. at $(u, v))$. It is natural to ask if this is due that the fact that the fiber of $f$ containing $S$ (resp. $(u, v))$ has a positive-dimensional component passing through $S$ (resp. $(u, v)$ ). Easy examples shows that this is not always the case (Examples 10 and 11).

Example 10. Fix integer $r \geq 3$. We claim the existence of a smooth, rational and non-degenerate curve $X \subset \mathbb{P}^{r}$ and $u, v \in X$ such that $u \neq v, T_{u} X=T_{c} X$ and $\operatorname{dim}\left\langle O(X, 2)_{u} \cup O(X, 2)_{v}\right\rangle=3$, i.e., $\operatorname{dim} O(X, 2)_{u}=\operatorname{dim} O(X, 2)_{v}=2$ and $O(X, 2)_{u} \cap O(X, 2)_{v}=T_{u} X$. In this case the fiber of the abstract 2-secant map of $X$ at $(u, v)$ is finite. Fix an integer $d \geq 7$. Let $C \subset \mathbb{P}^{d}$ be the rational normal curve. Fix $o, p \in C$ such that $o \neq p$. Since $C$ is a rational normal curve and $d \geq 7$, the degree 6 scheme $(3 o, C) \cup(3 p, C)$ is linearly independent. Thus, $\operatorname{dim}\langle(2 o, C) \cup(2 p, C)\rangle=3$. Fix a general line $L \subset\langle(2 o, C) \cup(2 p, C)\rangle$ and a general linear subspace $M \subset \mathbb{P}^{d}$ such that $\operatorname{dim} M=d-r-1$ and $L \subseteq M$. Since $r \geq 3$ and $M$ is general, $M \cap\langle(3 o, C) \cup(3 p, C)\rangle=L$.

Claim 1: $M \cap \sigma_{2}(C)=\varnothing$ and the linear projection $\ell: \mathbb{P}^{d} \backslash M \rightarrow \mathbb{P}^{r}$ from $M$ induces an isomorphism between $C$ and the degree d curve $X:=\ell(C) \subset \mathbb{P}^{r}$.

Proof of Claim 1: Since $C$ is smooth, each point of $\sigma_{2}(C)$ is contained in a tangent line or a secant line of $C$. Since $C \subset \mathbb{P}^{d}$ is a rational normal curve, every closed subscheme of $C$ of degree at most $d+1$ is linearly independent. Thus, the assumption $d \geq 7$ implies $\sigma_{2}(C) \cap\langle(3 o, C) \cup(3 p, C)\rangle=(2 o, C) \cup(2 p, C)$. Since $L$ is general in $\langle(2 o, C) \cup(2 p, C)\rangle$ and $M$ has codimension at least 3 in $\mathbb{P}^{d}, M \cap \sigma_{2}(C)=\varnothing$ and hence $M \cap C=\varnothing$ and $\ell_{\mid C}$ induces an isomorphism between $C$ and the degree $d$ non-degenerate curve $X$.

Claim 1 shows that $\operatorname{dim} O(X, 2)_{u}=\operatorname{dim} O(X, 2)_{v}=2$ and $O(X, 2)_{u} \cap O(X, 2)_{v}=T_{u} X$. Since $\operatorname{dim} X=1$, the fibers of the map $\pi: \Sigma_{2}^{0}(X) \rightarrow \sigma_{2}(X)$ have dimension 0 .

Example 11. Fix integer $r \geq 4$. We claim the existence of a smooth, rational and non-degenerate curve $X \subset \mathbb{P}^{r}$ and $u, v \in X$ such that $u \neq v, T_{u} X \neq T_{v} X$ is a point not in $X$ and $\operatorname{dim}\left\langle O(X, 2)_{u} \cup\right.$ $\left.O(X, 2)_{v}\right\rangle=4$, i.e., $\operatorname{dim} O(X, 2)_{u}=\operatorname{dim} O(X, 2)_{v}=2$ and $O(X, 2)_{u} \cap O(X, 2)_{v}=T_{u} X \cap$ $T_{v} X$. In this case the fiber of the abstract 2-secant map of $X$ at $(u, v)$ is finite. Fix an integer $d \geq 8$. Let $C \subset \mathbb{P}^{d}$ be the rational normal curve. We adapt Example 10. Instead of $L$ we take a general $c \in\left\langle T_{o} C \cup T_{p} C\right\rangle$ and take as $M$ a general linear subspace of codimension $d-r-1$ containing $c$.

Fix an integral and non-degenerate $n$-dimensional variety $X \subset \mathbb{P}^{r}$ and a positive integer $s$ such that $\sigma_{s}(X) \subsetneq \mathbb{P}^{r}$. Let $\sigma_{s}^{00}(X)$ denote the set of all $q \in \mathbb{P}^{r}$ with $X$-rank $r$. Let $\sigma_{S}^{00}(X)$ uni denote the set of all $q \in \sigma_{s}^{00}(X)$ which are in the linear span of a unique subset of $X$ with cardinality. The sets $\sigma_{s}^{00}(X)$ and $\sigma_{s}^{00}(X)_{\text {uni }}$ are constructible and the first one contains a non-empty open subset of $\sigma_{S}(X)$. Assume that $\sigma_{S}(X)$ has the expected dimension, $s(n+1)-1$, to hope to have $\sigma_{s}^{00}(X)_{\text {uni }} \neq \varnothing$. There are criterion which guarantee that $\sigma_{S}^{00}(X)_{\text {uni }}$ is dense in $\sigma_{S}(X)$, (for any $X$ weak nondefectivity and tangential nondefectivity ([10-12]), for tensors and partially symmetric tensors the famous Kruskal criterion and its modifications; up to now for tensors the best results are in [37]).

Question 4. Assume $\sigma_{s}^{00}(X)_{\text {uni }}$ dense in $\sigma_{s}^{00}(X)$. Describe in specific cases the non-uniqueness set $\sigma_{S}^{00}(X) \backslash \sigma_{S}^{00}(X)$ uni.

There are examples with $\sigma_{s}(X)=\mathbb{P}^{r}, \sigma_{S}^{00}(X)$ containing a general $q \in \mathbb{P}^{r}, \sigma_{S}^{00}(X)_{\text {uni }} \neq$ $\varnothing$ and $\sigma_{S}^{00}(X)$ uni very small (linearly normal embeddings of elliptic curves). If $z:=r_{X}(q)<$ $r$, say $q=\langle S\rangle$ with $S \subset X$ and $\# S=z=1, q$ is in the linear span of all $S \cup\{o\}, o \in S \backslash\{o\}$, but none of these sets irredundantly spans $q$. For tensors a general tensor of each rank $s$ is irredundantly spanned by sets of $t$ points if $s<t \leq s$ in infinitely many ways ([38], Theorem 3.8). The following example with $z$ any positive integer shows that sometimes above its rank $z:=r_{X}(q)$ a point $q$ may be irredundantly spanned by a unique set of cardinality $z+1$. 
Example 12. We first construct a smooth curve $X \subset \mathbb{P}^{r}, r \geq 3$, and $q \in X$ such that there is a unique $S \subset X \backslash\{q\}$ with $\# S=2$ and $q \in\langle S\rangle$. Let $C \subset \mathbb{P}^{d} r+1$ be a rational normal curve of degree $r+1$. Fix 3 distinct points $a, b$ and $c$ of $C$ and let o be a general element of $\langle\{a, b, c\}\rangle$. Let $\ell_{0}: \mathbb{P}^{r+1} \backslash \mathbb{P}^{r}$ denote the linear projection from o. By Sylvester theorem (or because any subscheme of $C$ of degree $\leq d+1$ is linearly independent) there is no $Z \subset C$ such that $\operatorname{deg}(Z) \leq 2$ and $o \in\langle Z\rangle$. Thus, $o \notin C$ and $\ell=\ell_{o \mid C}$ induces an embedding of $C$ into $\mathbb{P}^{r}$. Set $X:=\ell(C), q:=\ell(a)$ and $S:=\ell(\{b, c\})$. Thus, $q \in X$ and the set $S$ irredundantly spans $q$. Assume the existence of a set $S^{\prime} \subset X$ such that $\# S^{\prime}=2$ and $S^{\prime}$ irredundantly spans q, i.e., $q \notin S^{\prime}$ and $q \in\left\langle S^{\prime}\right.$. Set $E:=\ell^{-1}\left(S^{\prime}\right)$ and $F:=\{a, b, c\} \cup E$. Since $\ell$ is an embedding, $\# F=5$. Since $r+1 \geq 4, \operatorname{dim}\langle F\rangle=4$. Thus, $\operatorname{dim} \ell_{o}(\langle F\rangle \backslash\{o\})=3$ contradicting the fact that the lines $\langle S\rangle$ and $\left\langle S^{\prime}\right\rangle$ meets at $q$. Now we fix an integer $z>1$ and modify the previous example to obtain one for $z$. Fix an integer $r \geq 3 z$. Let $C \subset \mathbb{P}^{r+1}$ be a rational normal curve. Fix $2 z+1$ general points $a_{1}, \ldots, a_{z}, b_{1}, \ldots, b_{z+1}$ and a general $o \in\left\langle\left\{a_{1}, \ldots, a_{z}, b_{1}, \ldots, b_{z+1}\right\}\right\rangle$. Since $r \geq 3 z$, any closed subscheme of $C$ of degree $\leq 3 z+2$ is linearly independent. Let $\ell_{0}: \mathbb{P}^{r+1} \backslash \mathbb{P}^{r}$ denote the linear projection from 0 . The map $\ell_{0}$ induces an embedding $\ell: C \rightarrow \mathbb{P}^{r+1}$. Set $X:=\ell(C)$. By construction the $(z-1)$-dimensional space $\left\langle\ell\left(\left\{a_{1}, \ldots, a_{z}\right\}\right)\right\rangle$ meets the $z$-dimensional space $\left\langle\ell\left(\left\{b_{1}, \ldots, b_{z+1}\right\}\right)\right\rangle$ at a unique point, $q$. Since any $3 z+2$ points of $C$ are linearly independent, we first see that $q$ has $X$-rank $z$, then that $\ell\left(\left\{a_{1}, \ldots, a_{z}\right\}\right)$ is the unique set evincing the $X$-rank of $q$ and then that $\ell\left(\left\{b_{1}, \ldots, b_{z+1}\right\}\right)$ is the unique subset of $X$ with cardinality $z+1$ irredundantly spanning $q$.

Funding: This research received no external funding.

Institutional Review Board Statement: Not applicable.

Informed Consent Statement: Not applicable.

Conflicts of Interest: The author declares no conflict of interest.

\section{References}

1. Ådlandsvik, B. Joins and higher secant varieties. Math. Scan. 1987, 61, 213-222. [CrossRef]

2. Ballico, E.; Bernardi, A.; Santarsiero, P. Terracini loci for 3 points on a Segre variety. arXiv 2020, arXiv:2012.00574.

3. Ballico, E.; Chiantini, L. On the Terracini locus of projective varieties. Milan J. Math. 2021, 89, 1-17. [CrossRef]

4. Chandler, K. Hilbert functions of dots in linear general position. In Proceedings of the Conference on Zero-Dimensional Schemes, Ravello, Italy, 7 June 1992; pp. 65-79.

5. Chandler, K.A. Geometry of dots and ropes. Trans. Am. Math. Soc. 1995, 347, 767-784. [CrossRef]

6. Chandler, K.A. A brief proof of a maximal rank theorem for generic double points in projective space. Trans. Am. Math. Soc. 2000, 353, 1907-1920. [CrossRef]

7. Ballico, E. On the irreducibility of the Severi variety of nodal curves in a smooth surface. Arch. Math. 2019, 113, 483-487. [CrossRef]

8. Dedieu, T. Comment on: On the irreducibility of the Severi variety of nodal curves in a smooth surface, by E. Ballico. Arch. Math. 2020, 114, 171-174. [CrossRef]

9. Eastwood, A. Collision the biais et application à l'interpolation. Manuscripta Math. 1990, 67, 227-249. [CrossRef]

10. Chiantini, L.; Ciliberto, C. Weakly defective varieties. Trans. Am. Math. Soc. 2002, 454, 151-178. [CrossRef]

11. Chiantini, L.; Ciliberto, C. On the concept of $k$-secant order of a variety. J. Lond. Math. Soc. 2006, 73, 436-454. [CrossRef]

12. Chiantini, L.; Ciliberto, C. On the dimension of secant varieties. J. Eur. Math. Soc. 2006, 73, 436-454. [CrossRef]

13. Chiantini, L.; Ottaviani, G.; Vanniuwenhoven, N. On identifiability of symmetric tensors of subgeneric rank. Trans. Am. Math. Soc. 2017, 369, 4021-4042. [CrossRef]

14. Alexander, J.; Hirschowitz, A. Un lemme d'Horace différentiel: Application aux singularité hyperquartiques de $\mathbb{P}^{5}$. J. Algebr. Geom. 1992, 1, 411-426.

15. Alexander, J.; Hirschowitz, A. La méthode d'Horace éclaté: Application à l'interpolation en degré quatre. Invent. Math. 1992, 107, 585-602. [CrossRef]

16. Alexander, J.; Hirschowitz, A. Polynomial interpolation in several variables. J. Algebr. Geom. 1995, 4, $201-222$.

17. Brambilla, M.C.; Ottaviani, G. On the Alexander-Hirschowitz Theorem. J. Pure Appl. Algebra 2008, 212, 1229-1251. [CrossRef]

18. Hirschowitz, A. Le mèthod d'Horace. Manuscripta Math. 1985, 50, 337-388. [CrossRef]

19. Ciliberto, C.; Miranda, R.. Interpolations on curvilinear schemes. J. Algebra 1998, 203, 677-678. [CrossRef]

20. González, S.; Mallavibarrena, R. Osculating degeneration of curves. Comm. Algebra 2003, 31, 3829-3845. [CrossRef]

21. Ohno, M. An affirmative answer to a question of Ciliberto. Manuscripta Math. 1993, 81, 437-443. [CrossRef] 
22. Bolognesi, M.; Pirola, G. Osculating spaces and diophantine equations (with an Appendix by P. Corvaja and U. Zannier). Math. Nachr. 2011, 284, 960-972. [CrossRef]

23. Kaji, H. On the tangentially degenerate curves, II. Bull. Braz. Math. Soc. 2014, 45, 745-752. [CrossRef]

24. Kaji, H. On the tangentially degenerate curves. J. Lond. Math. Soc. 1986, 33, 430-440. [CrossRef]

25. Landsberg, J.M. Tensors: Geometry and Applications. 2012; Volume 128. Available online: http://213.230.96.51: 8090 / files / ebooks / Matematika/Landsberg\%20J.M.\%20Tensors..\%20geometry\%20and\%20applications\%20(GSM128,\%20 AMS, \%202012)(ISBN\%209780821869079)(O)(464s)\%20MAl\%20.pdf (accessed on 15 October 2021).

26. Ballico, E.; Bernardi, A.; Santarsiero, P. dentifiability of rank-3 tensors, Mediterranean. J. Math. 2021, 18, 1-26

27. Buczyński, J.; Landsberg, J.M. Ranks of tensors and a generalization of secant varieties. Linear Algebra Appl. 2013, 438, 668-689. [CrossRef]

28. Buczyński, J.; Landsberg, J.M. On the third secant variety. J. Algebr. Comb. 2014, 40, 475-502. [CrossRef]

29. Ballico, E. Linear dependent subsets of Segre varieties. J. Geom. 2020, 111, 23. doi: 10.1007/s00022-020-00534-7 [CrossRef]

30. Ballico, E. Linearly dependent and concise subsets of a Segre variety depending on $k$ factors. Bull. Korean Math. Soc. 2021, 58, 253-267. [CrossRef]

31. Ballico, E. Curvilinear subschemes of Segre varieties and the cactus rank. Gulf. J. Math. 2022, in press.

32. Hartshorne, R. Algebraic Geometry; Springer: New York, NY, USA, 1977.

33. Ballico, E.; Bernardi, A. Stratification of the fourth secant variety of Veronese variety via the symmetric rank. Adv. Pure Appl. Math. 2013, 4, 215-250. [CrossRef]

34. Abo, G. Ottaviani and C. Peterson, Induction for secant varieties of Segre varieties. Trans. Am. Math. Soc. 2006, 361, 767-792. [CrossRef]

35. Catalisano, M.V.; Geramita, A.V.; Gimigliano, A. Ranks of tensors, secant varieties of Segre varieties and fat points. Linear Algebra Appl. 2002, 355, 263-285. [CrossRef]

36. Catalisano, M.V.; Geramita, A.V.; Gimigliano, A. Higher secant varieties of the Segre varieties $\mathbb{P}^{1} \times \cdots \times \mathbb{P}^{1}$. J. Pure Appl. Algebra 2005, 201, 367-380. [CrossRef]

37. Lovitz, B.; Petrov, F. A generalization of Kruskal's theorem on tensor decomposition. arXiv 2021, arXiv:2103.15633.

38. Ballico, E.; Bernardi, A.; Chiantini, L.; Guardo, E. Bounds on the tensor rank. Ann. Mat. Pura Appl. 2018, 197, 1771-1785. [CrossRef] 